\title{
A LAGRANGE-TYPE PROJECTOR ON THE REAL LINE
}

\author{
G. MASTROIANNI AND I. NOTARANGELO
}

Dedicated to Professor J. Szabados on the occasion of his 70th birthday

\begin{abstract}
We introduce an interpolation process based on some of the zeros of the $m$ th generalized Freud polynomial. Convergence results and error estimates are given. In particular we show that, in some important function spaces, the interpolating polynomial behaves like the best approximation. Moreover the stability and the convergence of some quadrature rules are proved.
\end{abstract}

\section{INTRODUCTION}

In this paper we study the approximation of functions $f: \mathbb{R} \rightarrow \mathbb{R}$, by means of Lagrange interpolation, in some suitable weighted $L^{p}$-spaces. To be more precise, we consider functions increasing exponentially for $|x| \rightarrow \infty$ and having a singular inner point, which we assume to be 0 .

Accordingly, we introduce a weight of the form

$$
u(x)=|x|^{\gamma}(1+|x|)^{\mu} \mathrm{e}^{-\frac{|x|^{\lambda}}{2}}, \quad \gamma \geq 0,
$$

and we investigate the convergence of the interpolation process in $L_{u}^{p}$, for $1 \leq p \leq$ $\infty$. We remark that, since $u(0)=0$, the singularity of the function at 0 generates further difficulties and requires a more careful study of the polynomial behaviour in suitable neighborhoods of the origin (see for instance [23, 24, 25, 1, for the case $[-1,1]$, and more recent papers [21, 22] for the case of unbounded domains). Lemma $\mathrm{A}$ in Section 2 answers this problem.

In order to introduce our interpolation process, we consider the weight $w(x)=$ $|x|^{\alpha} \mathrm{e}^{-|x|^{\lambda}}$ and the corresponding sequence $\left\{p_{m}(w)\right\}_{m \in \mathbb{N}}$ of orthonormal polynomials with positive leading coefficients. Unfortunately, the behaviour of the projectors related to $\left\{p_{m}(w)\right\}_{m \in \mathbb{N}}$ is poor. For instance, in [19], the corresponding Fourier sums $\mathcal{S}_{m}(w, f)$ have been considered for functions $f \in L_{u}^{p}$ and, similarly to the cases of Freud and Laguerre systems, it has been proved that the inequality $\left\|\mathcal{S}_{m}(w, f) u\right\|_{p} \leq \mathrm{C}\|f u\|_{p}$, with $\mathrm{C}>0$ a constant, holds true only if $p \in(4 / 3,4)$. Furthermore, the Lagrange polynomials $\mathcal{L}_{m}(w, f)$ interpolating $f$ at the zeros of $p_{m}(w)$ behave as those based on Freud zeros.

Received by the editor March 31, 2008 and, in revised form, March 23, 2009.

2000 Mathematics Subject Classification. Primary 41A05, 65D05, 65D30, 65D32; Secondary $41 \mathrm{~A} 10$.

Key words and phrases. Orthogonal polynomials, Lagrange interpolation, quadrature rules.

This research was partially supported by Ministero dell'Università e della Ricerca, PRIN 2006 "Numerical methods for structured linear algebra and applications".

(C)2009 American Mathematical Society Reverts to public domain 28 years from publication 
To overcome such a problem, in [26, 17, 30] the authors have suggested to interpolate a "finite section" of the function and then to estimate a finite section of the interpolating polynomial in suitable weighted norms. A similar method has been used for Fourier sums in [26, 19]. To fix the idea, denoting by $a_{m}=$ $a_{m}(w)$ the Mhaskar-Rahmanov-Saff number of $w$ and by $\chi_{m}$ the characteristic function of the interval $\left[-\theta a_{m}, \theta a_{m}\right], 0<\theta<1$, the $L_{u}^{p}$-convergence of the sequences $\left\{\chi_{m} \mathcal{L}_{m}\left(w, \chi_{m} f\right)\right\}_{m \in \mathbb{N}}$ and $\left\{\chi_{m} \mathcal{S}_{m}\left(w, \chi_{m} f\right)\right\}_{m \in \mathbb{N}}$ has been proved in [26], for $1<p<\infty$ and $\alpha=\gamma=\mu=0$.

But $\chi_{m} \mathcal{L}_{m}\left(w, \chi_{m} f\right)$ is a "truncated" polynomial, and therefore $\chi_{m} \mathcal{L}_{m}(w)$ does not project a function belonging to $L_{u}^{\infty}$ into the space $\mathbb{P}_{m-1}$ of the polynomials of degree at most $m-1$. On the other hand, bounded projectors as well as those having the smallest norm are crucial tools in different contexts, for instance in the numerical treatment of functional equations.

In order to obtain these kinds of projectors, following an idea previously used in [33, 10, we denote by $L_{m+2}(w, f)$ the Lagrange polynomial interpolating $f \in L_{u}^{\infty}$ at the zeros of $p_{m}(w)$ and the extra points $\pm a_{m}$. Then we set $L_{m+2}^{*}(w, f)=$ $L_{m+2}\left(w, \chi_{j} f\right)$, where $f \in L_{u}^{\infty}$ and $\chi_{j}$ denotes the characteristic function of $\left[-x_{j}, x_{j}\right]$, with $x_{j}=x_{j(m)}=\min _{k}\left\{x_{k}: x_{k} \geq \theta a_{m}\right\}, \theta \in(0,1)$. The operator $L_{m+2}^{*}(w)$ does not project $L_{u}^{\infty}$ onto $\mathbb{P}_{m+1}$, but onto a subspace $\mathcal{P}_{m+1} \subset \mathbb{P}_{m+1}$. We prove that $\bigcup_{m} \mathcal{P}_{m+1}$ is dense in $L_{u}^{p}$, for $1 \leq p \leq \infty$, and that for each element of $\mathcal{P}_{m+1}$, both Marcinkiewicz inequalities hold true. As a by-product, we characterize the Marcinkiewicz bases in $\mathcal{P}_{m+1}$. Moreover, under simple necessary and sufficient conditions on the weights $w$ and $u$, we show that $L_{m+2}^{*}(w)$ is a uniformly bounded operator in some important subspace of $L_{u}^{p}$, with $1<p<\infty$.

Finally, using the same operator, we introduce a Gauss-type rule and a "product rule" and we prove their stability and convergence.

This paper is structured as follows. In Section 2 we recall some basic facts. In Section 3 we give our main results and in Section 4 we prove them.

\section{BASIC FACTS}

Function spaces. Let $v:[0,+\infty) \rightarrow \mathbb{R}^{+}$be a continuous and nondecreasing function with $v(0)=0$. Additionally assume that, for each $\varepsilon>0, v(x) / x^{\varepsilon}$ is nonincreasing and $\lim _{x \rightarrow 0^{+}}\left[v(x) / x^{\varepsilon}\right]=+\infty$. Simple examples of such kinds of weight functions are given by $v(x)=\log ^{\alpha} \log (\mathrm{e}+x)$ and $v(x)=\log ^{-\beta}(1+1 / x)$, with $\alpha, \beta>0$. Setting $\varrho(x)=|x|^{\gamma}(1+|x|)^{\mu} v(|x|), \gamma \geq 0, \mu \in \mathbb{R}$, we introduce the weight function

$$
u(x)=\varrho(x) \mathrm{e}^{-\frac{|x|}{2}}, \quad \lambda>1 .
$$

For $1 \leq p<\infty$ we denote by $L_{u}^{p}$ the set of all measurable functions $f$ such that

$$
\|f\|_{L_{u}^{p}}:=\|f u\|_{p}=\left(\int_{\mathbb{R}}|f(x) u(x)|^{p} \mathrm{~d} x\right)^{1 / p}<\infty,
$$

while for $p=\infty$ we introduce the space

$$
C_{u}:=L_{u}^{\infty}= \begin{cases}\left\{f \in C^{0}(\mathbb{R}): \lim _{x \rightarrow \pm \infty} f(x) u(x)=0\right\}, & \gamma=0, \\ \left\{f \in C^{0}(\mathbb{R} \backslash\{0\}): \lim _{x \rightarrow 0 \text { or } \pm \infty} f(x) u(x)=0\right\}, & \gamma>0,\end{cases}
$$


with the norm

$$
\|f\|_{L_{u}^{\infty}}:=\|f u\|_{\infty}=\sup _{x \in \mathbb{R}}|f(x) u(x)| .
$$

Such spaces $L_{u}^{p}$ with the above-defined norms are Banach spaces. Note that a function in $L_{u}^{p}$ does not belong necessarily to $L^{p}$ and that the Weierstrass theorem implies the limit conditions in the definition of $C_{u}$.

We remark that, by definition of $v$, if a weight of the form $|x|^{\sigma}(1+|x|)^{\tau}$ is integrable on $\mathbb{R}$, so is also $|x|^{\sigma}(1+|x|)^{\tau} v(|x|)$ (see for instance [20]).

Let us define the Sobolev spaces by

$$
W_{r}^{p}(u)=\left\{f \in L_{u}^{p}: f^{(r-1)} \in A C(\mathbb{R} \backslash\{0\}),\left\|f^{(r)} u\right\|_{p}<\infty\right\}, \quad r \geq 1,
$$

where $A C(\mathbb{R} \backslash\{0\})$ denotes the set of all the functions which are absolutely continuous on every closed subset of $\mathbb{R} \backslash\{0\}$. We equip these spaces with the norm

$$
\|f\|_{W_{r}^{p}(u)}=\|f u\|_{p}+\left\|f^{(r)} u\right\|_{p} .
$$

For any $f \in L_{u}^{p}, 1 \leq p \leq \infty$, we consider the following $r$ th $\left(r \in \mathbb{Z}^{+}\right)$modulus of smoothness (this definition appeared in [21, 22])

$$
\omega^{r}(f, t)_{u, p}^{*}=\Omega^{r}(f, t)_{u, p}^{*}+\sum_{k=1}^{3} \inf _{P \in \mathbb{P}_{r-1}}\|(f-P) u\|_{L^{p}\left(\mathcal{I}_{k}\right)},
$$

with step $t<t_{0}\left(t_{0}\right.$ sufficiently small $), \mathcal{I}_{1}=\left(-\infty,-\mathrm{A} r t^{-1 /(\lambda-1)}\right), \mathcal{I}_{2}=(-4 r t, 4 r t)$ and $\mathcal{I}_{3}=\left(\operatorname{Art} t^{-1 /(\lambda-1)},+\infty\right)$. Its main part $\Omega^{r}(f, t)_{u, p}^{*}$ is defined by

$$
\Omega^{r}(f, t)_{u, p}^{*}=\sup _{0<h \leq t}\left\|\Delta_{h}^{r}(f) u\right\|_{L^{p}\left(\mathcal{I}_{r, h}\right)},
$$

where $\mathcal{I}_{r, h}=\left[-\mathrm{A} r h^{-1 /(\lambda-1)},-4 r h\right] \cup\left[4 r h, \mathrm{~A} r h^{-1 /(\lambda-1)}\right], \mathrm{A}>0$ is a constant and

$$
\Delta_{h} f(x)=f\left(x+\frac{h}{2}\right)-f\left(x-\frac{h}{2}\right), \quad \Delta^{r}=\Delta\left(\Delta^{r-1}\right) .
$$

In the sequel, $\mathrm{C}$ will stand for a positive constant that could assume different values in each formula and we shall write $\mathrm{C} \neq \mathrm{C}(a, b, \ldots)$ when $\mathrm{C}$ is independent of $a, b, \ldots$ Furthermore $A \sim B$ will mean that if $A$ and $B$ are positive quantities depending on some parameters, then there exists a positive constant $\mathrm{C}$ independent of these parameters such that $(A / B)^{ \pm 1} \leq \mathrm{C}$.

Let us denote by $\mathbb{P}_{m}$ the set of all algebraic polynomials of degree at most $m$ and by $E_{m}(f)_{u, p}=\inf _{P \in \mathbb{P}_{m}}\|(f-P) u\|_{p}$ the error of best polynomial approximation in $L_{u}^{p}$. In 21, 22] the following Jackson and Stechkin-type inequalities have been proved:

$$
\begin{gathered}
E_{m}(f)_{u, p} \leq \mathrm{C} \omega^{r}\left(f, \frac{a_{m}}{m}\right)_{u, p}^{*}, \quad r<m, \\
\omega^{r}\left(f, \frac{a_{m}}{m}\right)_{u, p}^{*} \leq \mathrm{C}\left(\frac{a_{m}}{m}\right)^{r} \sum_{k=1}^{\left\lfloor\frac{m}{a_{m}}\right\rfloor}\left(\frac{k}{a_{k}}\right)^{r} \frac{E_{k}(f)_{u, p}}{k},
\end{gathered}
$$

where $f \in L_{u}^{p}, 1 \leq p \leq \infty, a_{m} \sim m^{1 / \lambda}$ is the Mhaskar-Rahmanov-Saff (M-R-S, for short) number related to the weight $u$; in both cases, $\mathrm{C}$ is independent of $f$ and $m$, and $\lfloor a\rfloor$ stands for the largest integer smaller than $a \in \mathbb{R}^{+}$or equal. 
An estimate, weaker than (2.2), for the error of best polynomial approximation is given by

$$
\begin{aligned}
E_{m}(f)_{u, p} & \leq \mathrm{C} \int_{0}^{\frac{a_{m}}{m}}\left[\Omega^{r}(f, t)_{u, p}^{*}+\inf _{P \in \mathbb{P}_{r-1}}\|(f-P)\|_{L^{p}(-4 r t, 4 r t)}\right] \frac{\mathrm{d} t}{t} \\
& =\mathrm{C} \int_{0}^{\frac{a_{m}}{m}} \frac{A^{r}(f, t)_{u, p}}{t} \mathrm{~d} t,
\end{aligned}
$$

where $1 \leq p \leq \infty$ and $\mathrm{C} \neq \mathrm{C}(m, f)$. Inequality (2.4) can be deduced from [22] (see also 2 $)$. In order to estimate the second addend in (2.4), we can use the following lemma, proved in [1, 22.

Lemma A. Let $f \in W_{r}^{p}(u), 1 \leq p \leq \infty, r \geq 1, \gamma>0$. If $\gamma+1 / p>r$, then

$$
\|f u\|_{L^{p}(-t, t)} \leq \mathrm{C} t^{r}\left(\|f u\|_{L^{p}(-a, a)}+\left\|f^{(r)} u\right\|_{L^{p}(-a, a)}\right), \quad a>t .
$$

While if $\gamma+1 / p \leq r, \gamma+1 / p \neq 1,2, \ldots, r$ and $f^{r-1-\tau}(0)$ exists, with $\tau=\lfloor\gamma+1 / p\rfloor$, then there exists a polynomial $P \in \mathbb{P}_{r-1-\tau}$ such that

$$
\|(f-P) u\|_{L^{p}(-t, t)} \leq \mathrm{C} t^{r}\left(\|f u\|_{L^{p}(-a, a)}+\left\|f^{(r)} u\right\|_{L^{p}(-a, a)}\right) .
$$

In both cases, $\mathrm{C}$ is independent of $f$ and $t$.

To complete Lemma A, we remark that if $\gamma+1 / p=r$, then (2.5) holds true with $t^{r} \log t^{-1}$ in place of $t^{r}$ and if $\gamma+1 / p=\nu \in \mathbb{N}, \nu<r$, then (2.6) holds true with $\nu$ and $t^{r} \log t^{-1}$ in place of $\tau$ and $t^{r}$, respectively.

For simplicity, in the sequel we will assume that $\gamma+1 / p$ is not an integer.

By means of the modulus of smoothness, for $1 \leq p \leq \infty$, we can define the Zygmund spaces

$$
Z_{s}^{p}(u)=\left\{f \in L_{u}^{p}: \sup _{t>0} \frac{\omega^{r}(f, t)_{u, p}^{*}}{t^{s}}<\infty, r>s\right\}, \quad s \in \mathbb{R}^{+},
$$

equipped with the norm

$$
\|f\|_{Z_{s}^{p}(u)}=\|f\|_{L_{u}^{p}}+\sup _{t>0} \frac{\omega^{r}(f, t)_{u, p}^{*}}{t^{s}} .
$$

We remark that, by inequalities (2.4) and (2.3), $\sup _{t>0} A^{r}(f, t)_{u, p} t^{-s}<\infty$ implies $\sup _{t>0} \omega^{r}(f, t)_{u, p}^{*} t^{-s}<\infty$. Therefore, in the definition of the Zygmund space, $\omega^{r}(f, t)_{u, p}^{*}$ can be replaced by $A^{r}(f, t)_{u, p}$.

Orthonormal polynomials and Lagrange-type projector. Let us consider the weight $w(x)=|x|^{\alpha} \mathrm{e}^{-|x|^{\lambda}}, \alpha>-1, \lambda>1$, and the related M-R-S number $a_{m}$, defined by (see for instance [11])

$$
a_{m}=a_{m}(w)=\left[\frac{2 \pi}{\lambda B\left(\frac{\lambda+1}{2}, \frac{1}{2}\right)}\right]^{1 / \lambda}\left(1+\frac{\alpha}{2 m}\right)^{1 / \lambda} m^{1 / \lambda},
$$

where $B$ is the beta function.

Let $\left\{p_{m}(w)\right\}_{m \in \mathbb{N}}$ be the corresponding sequence of orthonormal polynomials with positive leading coefficients. These polynomials, sometimes called generalized Freud polynomials, have been extensively studied in [5, 13, 28, 6, 7]. 
We denote by $x_{k}, 1 \leq k \leq\lfloor m / 2\rfloor$, the positive zeros of $p_{m}(w)$ and by $x_{-k}$ the negative ones. If $m$ is odd, then $x_{0}=0$ is a zero of $p_{m}(w)$. These zeros are located as follows:

$$
-a_{m}\left(1-\frac{\mathrm{C}}{m^{2 / 3}}\right) \leq x_{-\lfloor m / 2\rfloor}<\cdots<x_{1}<x_{2}<\cdots<x_{\lfloor m / 2\rfloor} \leq a_{m}\left(1-\frac{\mathrm{C}}{m^{2 / 3}}\right)
$$

where $\mathrm{C}$ is a positive constant independent of $m$ (see [13]).

In order to introduce a subspace of $\mathbb{P}_{m+1}$, we assume $m$ to be even and, for a fixed $\theta \in(0,1)$, we set

$$
x_{j}=\min _{1 \leq k \leq \frac{m}{2}}\left\{x_{k}: x_{k} \geq \theta a_{m}\right\} .
$$

So we call $\mathcal{P}_{m+1}$ the subspace of $\mathbb{P}_{m+1}$ defined by

$$
\mathcal{P}_{m+1}=\left\{Q \in \mathbb{P}_{m+1}: Q\left( \pm a_{m}\right)=Q\left(x_{i}\right)=0,|i|>j\right\}
$$

Setting

$$
\varphi_{k}(x)=\frac{\ell_{k}^{*}(w ; x)}{u\left(x_{k}\right)}, \quad|k| \leq j
$$

with

$$
\ell_{k}^{*}(w ; x)=\frac{p_{m}(w ; x)}{p_{m}^{\prime}\left(w ; x_{k}\right)\left(x-x_{k}\right)} \frac{\left(a_{m}^{2}-x^{2}\right)}{\left(a_{m}^{2}-x_{k}^{2}\right)},
$$

the collection $\left\{\varphi_{-j}, \ldots, \varphi_{-1}, \varphi_{1}, \ldots, \varphi_{j}\right\}$ is a basis of $\mathcal{P}_{m+1}$ and, therefore, every polynomial $Q \in \mathcal{P}_{m+1}$ can be written in a unique manner as

$$
Q(x)=\sum_{|k| \leq j} \varphi_{k}(x) Q\left(x_{k}\right) u\left(x_{k}\right)=: L_{m+2}^{*}(w, Q ; x) .
$$

Extending the operator $L_{m+2}^{*}(w)$ to the functions $f \in C_{u}$, we introduce

$$
L_{m+2}^{*}(w, f ; x)=\sum_{|k| \leq j} \ell_{k}^{*}(w ; x) f\left(x_{k}\right) .
$$

This polynomial interpolates the function $f$ at each zero $x_{k}(|k| \leq j)$, and it vanishes at the points $\pm a_{m}$ and $x_{i}(|i|>j)$. So it belongs to $\mathcal{P}_{m+1}$. Therefore $L_{m+2}^{*}(w)$ : $C_{u} \rightarrow \mathcal{P}_{m+1}$ is a projector.

Notice that the Lagrange polynomial, $L_{m+2}(w, f)$, interpolating $f$ at the knots $x_{i}(i \leq m / 2)$ and $\pm a_{m}(w)$, can be written as

$$
L_{m+2}(w, f ; x)=\sum_{|k| \leq \frac{m}{2}+1} \ell_{k}^{*}(w ; x) f\left(x_{k}\right),
$$

where $\ell_{k}^{*}(w)$ are defined as in (2.9) for $|k| \leq m / 2$ and

$$
\ell_{ \pm\left(\frac{m}{2}+1\right)}^{*}(w ; x)=\frac{a_{m} \pm x}{2 a_{m}} \frac{p_{m}(w ; x)}{p_{m}\left(w ; \pm a_{m}\right)} .
$$

Then $L_{m+2}^{*}(w, f)$ is not a "truncated" polynomial as in [26, 30, but it satisfies

$$
L_{m+2}^{*}(w, f)=L_{m+2}\left(w, f_{j}\right),
$$

where $f_{j}=\chi_{j} f$ and $\chi_{j}$ is the characteristic function of the interval $\left[-x_{j}, x_{j}\right]$.

Up to now we supposed $m$ to be even. Nevertheless, if $m$ is odd, we can replace $p_{m}(w ; x)$ by $p_{m}(w ; x)\left(x-\widetilde{x}_{0}\right) / x$, where $\widetilde{x}_{0} \neq 0$ is such that $x_{1}-\widetilde{x}_{0} \sim a_{m} / m$. For instance we can choose $\widetilde{x}_{0}=x_{1} / 2$ (see [30] for more details). So, without loss of generality, from now on we tacitly assume $m$ to be even. 
From a numerical point of view, in order to compute $L_{m+2}^{*}(w, f)$, we observe that if $\lambda=2$, i.e. $w(x)=|x|^{\alpha} \mathrm{e}^{-x^{2}}$, then $\left\{p_{m}(w)\right\}_{m \in \mathbb{N}}$ is the sequence of SoninMarkov polynomials, which are simply related to Laguerre polynomials (see [8]). In the case $\lambda \neq 2$ we can use the Mathematica Package "OrthogonalPolynomials" (see [4]).

The following Marcinkiewicz-type inequality holds true.

Lemma 2.1. Let $1 \leq p<\infty, u(x)=\varrho(x) \mathrm{e}^{-\frac{|x|^{\lambda}}{2}}, \lambda>1$. Let $x_{k}, k \leq m / 2$, be the zeros of $p_{m}(w)$ and set

$$
\Delta x_{k}= \begin{cases}x_{k+1}-x_{k}, & k>0, \\ x_{k}-x_{k-1}, & k<0 .\end{cases}
$$

For any polynomial $Q \in \mathbb{P}_{\mathrm{b} m}$, with $\mathrm{b}$ a fixed integer, there exists $\theta_{1} \in(\theta, 1)$ such that

$$
\sum_{|k| \leq j} \Delta x_{k}\left|Q\left(x_{k}\right)\right|^{p} u^{p}\left(x_{k}\right) \leq \mathrm{C} \int_{\mathcal{A}_{m}^{*}}|Q(x)|^{p} u^{p}(x) \mathrm{d} x,
$$

where $\mathcal{A}_{m}^{*}:=\left[-\theta_{1} a_{m},-x_{1}\right] \cup\left[x_{1}, \theta_{1} a_{m}\right], j$ and $\theta$ are defined in (2.8) and $\mathrm{C}$ is a positive constant independent of $m$ and $Q$.

If $u(x)=\mathrm{e}^{-x^{2} / 2}$ and $x_{k}$ are Hermite zeros, an inequality similar to (2.12) was first proved by P. Nevai in [29].

We will omit the proof of Lemma 2.1, because it follows by the same method as in [26, 30]. But we emphasize that (2.12) seems to be false for $j=m / 2$ and $Q \in \mathbb{P}_{\mathrm{b} m}$ (see [14, 15] for similar remarks).

\section{MAin RESUlts}

First of all we are going to show that $\bigcup_{m} \mathcal{P}_{m+1}$ is dense in $L_{u}^{p}, 1 \leq p \leq \infty$. To this end in the next lemma we will prove a stronger statement.

In order to state the lemma we need some notation. We say that $P_{M} \in \mathbb{P}_{M}$ is a "near best polynomial approximant" of $f \in L_{u}^{p}$ if $\left\|\left(f-P_{M}\right) u\right\|_{p} \leq \mathrm{C} E_{M}(f)_{u, p}$, with $\mathrm{C} \neq \mathrm{C}(m, f)$. Moreover, with $\theta$ and $j$ defined in (2.8), we choose

$$
M=\left\lfloor\left(\frac{\theta}{\theta+1}\right)^{\lambda} \frac{m}{2}\right\rfloor=: \mathrm{cm},
$$

with $0<\mathrm{c}<1$. Finally, we set

$$
\widetilde{E}_{m+1}(f)_{u, p}=\inf _{Q \in \mathcal{P}_{m+1}}\|(f-Q) u\|_{p} .
$$

Lemma 3.1. Suppose that $w$ and $u$ are the weights defined above with arbitrarily fixed parameters $\lambda>1, \alpha, \gamma, \mu$. Then, for every function $f \in L_{u}^{p}, 1 \leq p \leq \infty$, we have

$$
\widetilde{E}_{m+1}(f)_{u, p} \leq\left\|\left[f-L_{m+2}^{*}\left(w, P_{M}\right)\right] u\right\|_{p} \leq \mathrm{C}\left\{E_{M}(f)_{u, p}+\mathrm{e}^{-\mathrm{A} m}\|f u\|_{p}\right\}
$$

and

$$
\left(\frac{a_{m}}{m}\right)^{r}\left\|L_{m+2}^{*}\left(w, P_{M}\right)^{(r)} u\right\|_{p} \leq \mathrm{C}\left\{\omega^{r}\left(f, \frac{a_{m}}{m}\right)_{u, p}^{*}+\left(\frac{a_{m}}{m}\right)^{r}\|f u\|_{p}\right\},
$$

where $r \in \mathbb{Z}^{+}, M$ is defined by (3.1), and $\mathrm{C}$ and $\mathrm{A}$ are positive constants independent of $m$ and $f$. 
Therefore, by virtue of Lemma 3.1, every $f \in L_{u}^{p}$ can be approximated by means of elements of $\mathcal{P}_{m+1}$. Moreover if $P_{M} \in \mathbb{P}_{M}$ is a near best polynomial approximant of $f$, its projection in $\mathcal{P}_{m+1}$ has an analogous behaviour with respect to $f$.

Now we are able to study the interpolation process $\left\{L_{m+2}^{*}(w)\right\}_{m \in \mathbb{N}}$ in $C_{u}$.

Theorem 3.2. Let $w$ and $u$ be the weights defined above and assume that their parameters $\alpha, \gamma, \mu$ satisfy

$$
0 \leq \gamma-\frac{\alpha}{2} \leq 1 \quad \text { and } \quad 0 \leq \mu \leq 1
$$

Then, for every $f \in C_{u}$, we have

$$
\begin{aligned}
\left\|L_{m+2}(w, f) u\right\|_{\infty} & \leq \mathrm{C} \log m\|f u\|_{\infty}, \\
\left\|\left[f-L_{m+2}(w, f)\right] u\right\|_{\infty} & \leq \mathrm{C} \log m E_{m+1}(f)_{u, \infty}
\end{aligned}
$$

and

$$
\left\|\left[f-L_{m+2}^{*}(w, f)\right] u\right\|_{\infty} \leq \mathrm{C}\left\{\log m E_{M}(f)_{u, \infty}+\mathrm{e}^{-\mathrm{A} m}\|f u\|_{\infty}\right\},
$$

where $M=\mathrm{cm}, 0<\mathrm{c}<1$, is defined by (3.1), $\mathrm{C} \neq \mathrm{C}(m, f)$ and $\mathrm{A} \neq \mathrm{A}(m, f)$.

Hence, by virtue of a result due to P. Vértesi [34], $L_{m+2}(w): C_{u} \rightarrow \mathbb{P}_{m+1}$ and consequently $L_{m+2}^{*}(w): C_{u} \rightarrow \mathcal{P}_{m+1}$ are projectors having the smallest norm (as order), but $L_{m+2}^{*}(w)$ has the advantage of requiring only $\lfloor\mathrm{b} m\rfloor(0<\mathrm{b}<1)$ evaluations of the function $f$.

In the case $\lambda=2$ and $\alpha=\gamma=\mu=0$, Theorem 3.2 recovers a result due to J. Szabados (see [33]).

The following lemma states a converse inequality of (2.12) for polynomials belonging to $\mathcal{P}_{m+1}$. To this purpose we recall that $u(x)=|x|^{\gamma}(1+|x|)^{\mu} v(|x|) \mathrm{e}^{-|x|^{\lambda} / 2}$ and $w(x)=|x|^{\alpha} \mathrm{e}^{-|x|^{\lambda}}, \alpha>-1, \lambda>1$; moreover we denote by $x_{k}(|k| \leq m / 2)$ the zeros of $p_{m}(w)$ and by $\Delta x_{k}$ the distance between two consecutive zeros defined in (2.11).

Lemma 3.3. Let $1<p<\infty$ and $\mu \geq 0$. Then, for every polynomial $Q \in \mathcal{P}_{m+1}$, we have

$$
\|Q u\|_{p} \leq \mathrm{C}\left(\sum_{|k| \leq j} \Delta x_{k}\left|Q\left(x_{k}\right)\right|^{p} u^{p}\left(x_{k}\right)\right)^{1 / p}, \quad \mathrm{C} \neq \mathrm{C}(m, Q),
$$

if and only if

$$
-\frac{1}{p}<\gamma-\frac{\alpha}{2}<1-\frac{1}{p}-\mu .
$$

We remark that (3.9) implies $\mu<1$. Moreover, recalling Lemma 2.1, under the assumptions of Lemma 3.3 , the equivalence

$$
\|Q u\|_{p} \sim\left(\sum_{|k| \leq j} \Delta x_{k}\left|Q\left(x_{k}\right)\right|^{p} u^{p}\left(x_{k}\right)\right)^{1 / p}
$$

holds true for all polynomials $Q \in \mathcal{P}_{m+1}$.

If $j=m / 2$ and $Q \in \mathbb{P}_{m+1}$, equivalence 3.10 is in general false, as has been proved in [26] when $w(x)=\mathrm{e}^{-|x|^{\lambda}}$ and $u=\sqrt{w}$ (see also [16]).

We want to show an interesting consequence of Lemma 3.3 and equivalence (3.10). Let $u$ be a weight function in $(a, b),-\infty \leq a<b \leq+\infty$. A basis of 
$\mathbb{P}_{m},\left\{\psi_{1}, \ldots, \psi_{m}\right\}$, is said to be a Marcinkiewicz basis if, for every polynomial $q_{m}(x)=\sum_{i=1}^{m} \beta_{i} \psi_{i}(x)$, it satisfies

$$
\left(\sum_{i=1}^{m}\left|\beta_{i}\right|^{p}\right)^{1 / p} \sim\left\|q_{m} u\right\|_{L^{p}(a, b)} .
$$

These bases appear in different contexts. If $p=2$ they are the well-known Riesz's bases. In the numerical treatment of functional equations, they play a crucial rule in the estimates of the condition numbers of matrices, coming from the discretization of operators.

J. Marcinkiewicz was the first one who introduced these kinds of bases, in the case of trigonometric polynomials. In the algebraic case, the Marcinkiewicz bases, related to the interval $(-1,1)$ and to generalized Jacobi weights, were constructed in 9] and 20]. According to our knowledge, up to now in the literature there were no examples of Marcinkiewicz bases on $(-\infty,+\infty)$ and related to exponential weights.

Now, by Lemma 3.3 and by (3.10), we can deduce that the functions

$$
\psi_{k}(x)=\frac{\ell_{k}^{*}(w ; x)}{\left(\Delta x_{k}\right)^{1 / p} u\left(x_{k}\right)}, \quad|k| \leq j,
$$

form a Marcinkiewicz basis of $\mathcal{P}_{m+1}$ with respect to the weight $u$ if and only if (3.9) is satisfied.

A further consequence of Lemma 3.3 is given by the following corollary.

Corollary 3.4. Let $u$ be defined by (2.1) and $\sigma(x)=(1+|x|)^{\nu} u(x)$. Assume $1<p<\infty$ and $\nu>\frac{1}{p}$. If (3.9) is fulfilled, then, for any $f \in C_{\sigma}$, we get

$$
\left\|L_{m+2}^{*}(w, f) u\right\|_{p} \leq \mathrm{C}\|f \sigma\|_{\infty},
$$

where $\mathrm{C}$ is a positive constant independent of $m$ and $f$.

Now we want to show that $L_{m+2}^{*}(w, f)$ behaves like the best polynomial approximation in the Zygmund spaces $Z_{r}^{p}(u)$ and in the Sobolev spaces $W_{r}^{p}(u)$. We will prove that in these spaces the operator $L_{m+2}^{*}(w)$ is uniformly bounded (obviously if (3.9) is fulfilled). To this end the following lemma is crucial.

Lemma 3.5. Let $1<p<\infty$. Let $w$ and $u$ be defined as before and assume that $f \in L_{u}^{p}$ satisfies

$$
\Omega^{r}(f, t)_{u, p}^{*} t^{-1-1 / p} \in L^{1}(0,1) .
$$

Then (3.9) is equivalent to each of the following estimates:

$$
\left\|L_{m+2}^{*}(w, f) u\right\|_{p} \leq \mathrm{C}\left\{\|f u\|_{p}+\left(\frac{a_{m}}{m}\right)^{1 / p} \int_{0}^{\frac{a_{m}}{m}} \frac{\Omega^{r}(f, t)_{u, p}^{*}}{t^{1+1 / p}} \mathrm{~d} t\right\}
$$

and

$$
\begin{aligned}
& \left\|\left[f-L_{m+2}^{*}(w, f)\right] u\right\|_{p} \\
& \leq \mathrm{C}\left\{\omega^{r}\left(f, \frac{a_{m}}{m}\right)_{u, p}^{*}+\left(\frac{a_{m}}{m}\right)^{r}\|f u\|_{p}+\left(\frac{a_{m}}{m}\right)^{1 / p} \int_{0}^{\frac{a_{m}}{m}} \frac{\Omega^{r}(f, t)_{u, p}^{*}}{t^{1+1 / p}} \mathrm{~d} t\right\}
\end{aligned}
$$

with $\mathrm{C}$ a positive constant independent of $m$ and $f$. 
In particular, if $f \in Z_{r}^{p}(u)$ and $r>1 / p$, i.e. $\sup _{t>0} \omega^{k}(f, t)_{u, p}^{*} t^{-r}<\infty, k>r>$ $1 / p$, then by (3.14) we have

$$
\left\|\left[f-L_{m+2}^{*}(w, f)\right] u\right\|_{p} \leq \mathrm{C}\left(\frac{a_{m}}{m}\right)^{r}\|f\|_{Z_{r}^{p}(u)} .
$$

The following theorem shows the uniform boundedness of the operator $L_{m+2}^{*}(w)$ in $Z_{r}^{p}(u)$.

Theorem 3.6. Let $1<p<\infty$. Under the assumption (3.9), for every $f \in Z_{r}^{p}(u)$, $r>1 / p$, we have

$$
\left\|L_{m+2}^{*}(w, f)\right\|_{Z_{r}^{p}(u)} \leq \mathrm{C}\|f\|_{Z_{r}^{p}(u)} .
$$

Moreover, for every $f \in Z_{s}^{p}(u), s>r>1 / p$, we get

$$
\left\|f-L_{m+2}^{*}(w, f)\right\|_{Z_{r}^{p}(u)} \leq \mathrm{C}\left(\frac{a_{m}}{m}\right)^{s-r}\|f\|_{Z_{s}^{p}(u)},
$$

where in both cases, $\mathrm{C}$ is a positive constant independent of $m$ and $f$.

Concerning the behaviour of $L_{m+2}^{*}(w, f)$, with $f \in W_{r}^{p}(u)$, we will assume (in addition to (3.9)) that $\gamma+1 / p$ is not an integer. Then from (3.14) the inequality

$$
\left\|\left[f-L_{m+2}^{*}(w, f)\right] u\right\|_{p} \leq \mathrm{C}\left(\frac{a_{m}}{m}\right)^{r}\|f\|_{W_{r}^{p}(u)}
$$

follows.

Theorem 3.7. Let $1<p<\infty$, assume $\gamma+1 / p$ is not an integer and let (3.9) be fulfilled. Then, for every $f \in W_{r}^{p}(u)$, we have

$$
\left\|L_{m+2}^{*}(w, f)\right\|_{W_{r}^{p}(u)} \leq \mathrm{C}\|f\|_{W_{r}^{p}(u)} .
$$

Moreover, for every $f \in W_{s}^{p}(u), s>r$, we have

$$
\left\|f-L_{m+2}^{*}(w, f)\right\|_{W_{r}^{p}(u)} \leq \mathrm{C}\left(\frac{a_{m}}{m}\right)^{s-r}\|f\|_{W_{s}^{p}(u)},
$$

where $\mathrm{C}$ in both cases is a positive constant independent of $m$ and $f$.

We finally remark that, for $\alpha=0$, hypothesis (3.9) becomes $-1 / p<\gamma<1-$ $\mu-1 / p$, and thus all the previous results are true for a more restricted class of functions.

Quadrature rules. We want to establish two statements concerning the main quadrature rules. Let us first consider a Gauss-type rule defined as

$$
\int_{\mathbb{R}} f(x) w(x) \mathrm{d} x=\sum_{|k| \leq j} \lambda_{k}(w) f\left(x_{k}\right)+e_{m}(f),
$$

where $m$ is even, $w$ is the weight defined above, $x_{k}$ are the zeros of $p_{m}(w), \lambda_{k}(w)$ are the Christoffel numbers and $e_{m}(f)$ is the remainder term. Rules of the form (3.21) for Laguerre and Freud weights appeared for the first time in 17, 3, 18.

The rule (3.21) can be obtained by replacing the integrating function $f$ by $L_{m+2}^{*}(w, f)$ and using the ordinary Gaussian rule. Obviously $e_{m}(f)=0$ if $f \in$ $\mathcal{P}_{m+1}$. In the following proposition we give a simple estimate of the error, which can be useful in different contexts. 
Proposition 3.8. Let $f \in C_{\sigma}$, where $\sigma(x)=|x|^{\gamma}(1+|x|)^{\mu} v(|x|) \mathrm{e}^{-\mathrm{b}|x|^{\lambda}}, 0<\mathrm{b} \leq 1$. If $\int_{\mathbb{R}} w(x) \sigma^{-1}(x) \mathrm{d} x<\infty$, then

$$
\left|e_{m}(f)\right| \leq \mathrm{C}\left\{E_{M}(f)_{\sigma, \infty}+\mathrm{e}^{-\mathrm{A} m}\|f \sigma\|_{\infty}\right\},
$$

where $M=\mathrm{cm}, \mathrm{c} \in(0,1)$ is fixed, and $\mathrm{C}$ is a positive constant independent of $m$ and $f$.

In many applications the following "product rule" can be useful:

$$
\int_{\mathbb{R}} f(x) k(x, y) u(x) \mathrm{d} x=\sum_{|k| \leq j} A_{k}(y) f\left(x_{k}\right)+e_{m}^{*}(f)=: G_{m}(f, y)+e_{m}^{*}(f),
$$

where $e_{m}^{*}(f)$ is the remainder term, $u(x)=|x|^{\gamma}(1+|x|)^{\mu} \mathrm{e}^{-|x|^{\lambda} / 2}$ and

$$
A_{k}(y)=\int_{\mathbb{R}} \ell_{k}^{*}(w ; x) k(x, y) u(x) \mathrm{d} x,
$$

with $\ell_{k}^{*}(w)$ the fundamental polynomials of $L_{m+2}^{*}(w, f)$, defined in (2.9), $w(x)=$ $|x|^{\alpha} \mathrm{e}^{-|x|^{\lambda}}, x_{j}=\min _{1 \leq k \leq \frac{m}{2}}\left\{x_{k}: x_{k} \geq \theta a_{m}\right\}, \theta \in(0,1)$.

We will treat in a further work the construction of the coefficients $A_{k}(y)$, which depend on the parameters of the weights $u$ and $w$, and strongly on the form of the kernel $k$. Here, we want to assign the conditions under which the quadrature rule $G_{m}(f, y)$ converges to the integral uniformly with respect to the parameter $y$.

Theorem 3.9. Let us assume that $k: \mathbb{R}^{2} \rightarrow \mathbb{R}$ and the weights $u$ and $w$ satisfy:

$$
\sup _{x \in \mathbb{R}} \frac{\sqrt{w(x)}}{u(x)}<\infty
$$

and

$$
\sup _{y \in \mathbb{R}} \int_{\mathbb{R}} \frac{u(x)}{\sqrt{w(x)}}|k(x, y)|\left(1+\log ^{+}|x|+\log ^{+}|k(x, y)|\right) \mathrm{d} x<\infty,
$$

where $\log ^{+} x=\log (\max \{1, x\})$. Then, for every function $f$ such that $\|f u\|_{\infty}<\infty$, we have

$$
\sup _{m} \sup _{y \in \mathbb{R}}\left|G_{m}(f, y)\right| \leq \mathrm{C}\|f u\|_{\infty}, \quad \mathrm{C} \neq \mathrm{C}(f) .
$$

Moreover (3.27) implies

$$
\sup _{y \in \mathbb{R}} \int_{\mathbb{R}} \frac{u(x)}{\sqrt{w(x)}}|k(x, y)| \mathrm{d} x<\infty .
$$

Thus, under the assumptions of Theorem 3.9, for every function $f \in C_{u}$, we have

$$
\left|e_{m}^{*}(f)\right| \leq \mathrm{C} \widetilde{E}_{m+1}(f)_{u, \infty},
$$

and to estimate $\widetilde{E}_{m+1}(f)_{u, \infty}$, we can use Lemma 3.1.

Furthermore, we emphasize that if $k(x, y) \equiv 1$, then, by (3.28), inequality (3.27) is not true. However, Theorem 3.9 comes in useful for a wide class of kernels. For instance, let us consider a kernel of the form

$$
\widetilde{k}(x, y)=\frac{P(x, y)}{Q(x, y)},
$$


where

$$
\begin{aligned}
& P(x, y)=\sum_{h, i} c_{i}\left|x-t_{h}\right|^{\gamma_{h}}\left|y-\tau_{i}\right|^{\delta_{i}}, \\
& Q(x, y)=\sum_{\eta, \nu} d_{\nu}\left|x-a_{\eta}\right|^{\alpha_{\eta}}\left|y-b_{\nu}\right|^{\beta_{\nu}}>0
\end{aligned}
$$

with $c_{i}, d_{\nu}, \gamma_{h}, \delta_{i}, \alpha_{\eta}, \beta_{\nu}, t_{h}, \tau_{i}, a_{\eta}, b_{\nu} \in \mathbb{R}$ and $h, i, \eta, \nu \leq N \in \mathbb{N}$. In this case, Theorem 3.9 turns into the following corollary.

Corollary 3.10. Let $\widetilde{k}: \mathbb{R}^{2} \rightarrow \mathbb{R}$ be as in (3.29). Assume that the weights $w$ and $u$ satisfy (3.25). Then, for every $f$ such that $\|f u\|_{\infty}<\infty$, we get

$$
\sup _{y \in \mathbb{R}}\left|G_{m}(f, y)\right| \leq \mathrm{C}\|f u\|_{\infty}, \quad \mathrm{C} \neq \mathrm{C}(m, f),
$$

if and only if

$$
\sup _{y \in \mathbb{R}} \int_{\mathbb{R}} \frac{u(x)}{\sqrt{w(x)}}|\widetilde{k}(x, y)| \mathrm{d} x<\infty .
$$

\section{Proofs}

First of all we recall some known results. The following lemma states some polynomial inequalities, which can be found in [21] (see also [31], pp. 277-343).

Lemma B. Let $u(x)=|x|^{\gamma}(1+|x|)^{\mu} \mathrm{e}^{-\frac{|x|^{\lambda}}{2}}$, with $\lambda>1, \mu \in \mathbb{R}$ and $\gamma>-\frac{1}{p}$ if $1<p<\infty, \gamma \geq 0$ if $p=\infty$. Moreover let $a_{m}:=a_{m}(u)$ be the $M-R-S$ number related to $u$. Then for any polynomial $P \in \mathbb{P}_{m}$, we have

$$
\left\|P^{\prime} u\right\|_{p} \leq \mathrm{C} \frac{m}{a_{m}}\|P u\|_{p}
$$

and

$$
\|P u\|_{q} \leq \mathrm{C}\left(\frac{m}{a_{m}}\right)^{\frac{1}{p}-\frac{1}{q}}\|P u\|_{p}, \quad 1 \leq p<q \leq \infty,
$$

where $\mathrm{C}$ is a constant independent of $m$ and $P$ in both cases.

Moreover, for any fixed $\mathrm{d}>0$, there exists $\mathrm{C}=\mathrm{C}(\mathrm{d}) \neq \mathrm{C}(m, P)$ such that

$$
\|P u\|_{p} \leq \mathrm{C}\|P u\|_{L^{p}\left(\mathcal{A}_{m}\right)}
$$

where $\mathcal{A}_{m}=\left[-a_{m},-\mathrm{d} \frac{a_{m}}{m}\right] \cup\left[\mathrm{d} \frac{a_{m}}{m}, a_{m}\right]$. Finally, $\forall \delta>0$, the inequality

$$
\|P u\|_{L^{p}\left(\mathcal{B}_{m}\right)} \leq \mathrm{Ce}^{-\mathrm{A} m}\|P u\|_{p}
$$

holds with $\mathcal{B}_{m}=\left\{x \in \mathbb{R}:|x|>(1+\delta) a_{m}\right\}$, C and A positive constants independent of $m$ and $P$.

The following inequalities deal with the behaviour of the orthonormal polynomials and their zeros $x_{k}$. In 5 it has been proved that

$$
\sup _{x \in\left[-a_{m}, a_{m}\right]}\left|p_{m}(w ; x) \mathrm{e}^{-\frac{|x|^{\lambda}}{2}}\left(|x|+\frac{a_{m}}{m}\right)^{\alpha / 2} \sqrt[4]{a_{m}^{2}-x^{2}}\right| \sim 1
$$

and

$$
\left|p_{m}(w ; x)\right| \sqrt{w(x)} \leq \frac{\mathrm{C}}{\sqrt[4]{a_{m}^{2}-x^{2}+m^{-2 / 3} a_{m}^{2}}}, \quad x \in \mathcal{A}_{m}
$$


where $\mathcal{A}_{m}$ is the subset defined in Lemma $\mathrm{B}$ with $a_{m}(w)$ in place of $a_{m}(u)$. Moreover, the equivalence

$$
\frac{1}{\left|p_{m}^{\prime}\left(w, x_{k}\right)\right| \sqrt{w\left(x_{k}\right)}} \sim \Delta x_{k} \sqrt[4]{a_{m}^{2}-x_{k}^{2}+\frac{a_{m}^{2}}{m^{2 / 3}}}, \quad|k| \leq \frac{m}{2},
$$

holds. Finally, the distance between two consecutive zeros of $p_{m}(w)$, defined by (2.11) (setting $x_{\frac{m}{2}+1}=-x_{-\frac{m}{2}-1}=a_{m}(w)$ ), can be estimated by

$$
\Delta x_{k} \sim \frac{a_{m}^{2}}{m} \frac{1}{\sqrt{a_{m}^{2}-x_{k}^{2}+m^{-2 / 3} a_{m}^{2}}}, \quad|k| \leq \frac{m}{2},
$$

from which we have

$$
\Delta x_{k} \sim \frac{a_{m}}{m}, \quad|k| \leq j .
$$

Here $\mathrm{C}$ and the constants in " $\sim$ " are independent of $m$ and $k$.

Using the previous inequalities, we can estimate the fundamental Lagrange polynomials, defined by (2.9) and (2.10). By (4.5) and (4.6), if $|k| \leq \frac{m}{2}$, we get

$$
\begin{aligned}
\frac{\left|\ell_{k}^{*}(w ; x)\right| u(x)}{u\left(x_{k}\right)} & =\frac{\left|p_{m}(w ; x)\right|\left(a_{m}^{2}-x^{2}\right) u(x)}{\left|p_{m}^{\prime}\left(w ; x_{k}\right)\right|\left|x-x_{k}\right|\left(a_{m}^{2}-x_{k}^{2}\right) u\left(x_{k}\right)} \\
& \leq \mathrm{C} \frac{v(|x|)}{v\left(\left|x_{k}\right|\right)}\left|\frac{x}{x_{k}}\right|^{\gamma-\frac{\alpha}{2}}\left(\frac{1+|x|}{1+\left|x_{k}\right|}\right)^{\mu} \frac{\Delta x_{k}}{\left|x-x_{k}\right|}
\end{aligned}
$$

for $x \in \mathcal{A}_{m}$ and $x_{k} \neq x_{d}, x_{d}$ being a node closest to $x$. Furthermore, the following equivalence is well known (see [11, or [12, pp. 320-321]):

$$
\frac{\left|\ell_{d}^{*}(w ; x)\right| u(x)}{u\left(x_{d}\right)} \sim 1
$$

uniformly with respect to $d$ and $m$. Finally, by (4.9) and (4.6), with $x=a_{m}$ and $x_{d}=x_{\frac{m}{2}}$, it follows that

$$
\left|p_{m}\left(w ; a_{m}\right)\right| \sqrt{w\left(a_{m}\right)} \geq \mathrm{C} \sqrt{\frac{m^{1 / 3}}{a_{m}}} .
$$

By (4.10) we get

$$
\begin{aligned}
\frac{\left|\ell_{\frac{m}{2}+1}^{*}(w ; x)\right| u(x)}{u\left(a_{m}\right)} & \leq \frac{\left|p_{m}(w ; x)\right| \sqrt{w(x)}}{\left|p_{m}\left(w ; a_{m}\right)\right| \sqrt{w\left(a_{m}\right)}} \frac{v(|x|)}{v\left(a_{m}\right)}\left|\frac{x}{a_{m}}\right|^{\gamma-\frac{\alpha}{2}}\left(\frac{1+|x|}{1+a_{m}}\right)^{\mu} \\
& \leq \frac{\mathrm{C}}{\sqrt[4]{a_{m}^{2}-x^{2}+m^{-2 / 3} a_{m}^{2}}} \sqrt{\frac{a_{m}}{m^{1 / 3}}} \frac{v(|x|)}{v\left(a_{m}\right)}\left|\frac{x}{a_{m}}\right|^{\gamma-\frac{\alpha}{2}}\left(\frac{1+|x|}{1+a_{m}}\right)^{\mu} \\
& \leq \mathrm{C} \frac{v(|x|)}{v\left(a_{m}\right)}\left|\frac{x}{a_{m}}\right|^{\gamma-\frac{\alpha}{2}}\left(\frac{1+|x|}{1+a_{m}}\right)^{\mu},
\end{aligned}
$$

for $x \in \mathcal{A}_{m}$. An analogous inequality holds for $\ell_{-\frac{m}{2}-1}^{*}(w)$. 
Now, for $x \in \mathcal{A}_{m}$, the sum

$$
\begin{aligned}
S_{m}(\alpha, \gamma, \mu, x) & :=\sum_{|k| \leq \frac{m}{2}, k \neq d} \frac{v(|x|)}{v\left(\left|x_{k}\right|\right)}\left|\frac{x}{x_{k}}\right|^{\gamma-\frac{\alpha}{2}}\left(\frac{1+|x|}{1+\left|x_{k}\right|}\right)^{\mu} \frac{\Delta x_{k}}{\left|x-x_{k}\right|} \\
& +2 \frac{v(|x|)}{v\left(a_{m}\right)}\left|\frac{x}{a_{m}}\right|^{\gamma-\frac{\alpha}{2}}\left(\frac{1+|x|}{1+a_{m}}\right)^{\mu}
\end{aligned}
$$

can be estimated as follows:

$$
S_{m}(\alpha, \gamma, \mu, x) \leq \mathrm{C} \log m \quad \text { if } 0 \leq \gamma-\frac{\alpha}{2} \leq 1,0 \leq \mu \leq 1 ;
$$

otherwise

$$
S_{m}(\alpha, \gamma, \mu, x) \leq \mathrm{C} m^{\tau},
$$

for some $\tau>0$ and $\alpha, \gamma, \mu$ arbitrarily fixed.

Proof of Lemma 3.1. We are going to prove (3.2) and (3.3) only for $1 \leq p<\infty$, the case $p=\infty$ being simpler. Let $P_{M} \in \mathbb{P}_{M}$ be a near best polynomial approximant of $f \in L_{u}^{p}, M=\left\lfloor\left(\frac{\theta}{\theta+1}\right)^{\lambda} \frac{m}{2}\right\rfloor$ and $Q=L_{m+2}^{*}\left(w, P_{M}\right)$. Using (4.3), we have

$$
\begin{aligned}
\widetilde{E}_{m+1}(f)_{u, p} & \leq\|(f-Q) u\|_{p} \\
& \leq\left\|\left(Q-P_{M}\right) u\right\|_{p}+\mathrm{C} E_{M}(f)_{u, p} \\
& =\left\|\left[L_{m+2}^{*}\left(w, P_{M}\right)-L_{m+2}\left(w, P_{M}\right)\right] u\right\|_{p}+\mathrm{C} E_{M}(f)_{u, p} \\
& \leq \mathrm{C}\left\|\left[L_{m+2}^{*}\left(w, P_{M}\right)-L_{m+2}\left(w, P_{M}\right)\right] u\right\|_{L^{p}\left(\mathcal{A}_{m}\right)}+\mathrm{C} E_{M}(f)_{u, p} .
\end{aligned}
$$

The first addend is dominated by

$$
\mathrm{C} a_{m}(u)^{1 / p} \sup _{|x| \geq \theta a_{m}(w)}\left|P_{M}(x) u(x)\right| \sup _{x \in \mathcal{A}_{m}} \sum_{|k| \leq \frac{m}{2}+1} \frac{\left|\ell_{k}^{*}(w ; x)\right| u(x)}{u\left(x_{k}\right)},
$$

where $\mathcal{A}_{m}=\left[-a_{m}(u),-\mathrm{d} \frac{a_{m}(u)}{m}\right] \cup\left[\mathrm{d} \frac{a_{m}(u)}{m}, a_{m}(u)\right], \mathrm{d}>0$. Using (4.9) and (4.13) the sum is not greater than $\mathrm{C} m^{\tau}$ for some $\tau>0$. Therefore it remains to estimate the quantity

$$
\gamma_{m}:=a_{m}(u)^{1 / p} m^{\tau} \sup _{|x| \geq \theta a_{m}(w)}\left|P_{M}(x) u(x)\right| .
$$

But, for the choice of $M$, it is easily seen that $\theta a_{m}(w) \geq(1+\theta) a_{M}(u)$. Then, using (4.4) and (4.2), we get

$$
\gamma_{m} \leq \mathrm{Ce}^{-\mathrm{A} m}\left\|P_{M} u\right\|_{p} \leq \mathrm{Ce}^{-\mathrm{A} m}\|f u\|_{p},
$$

from which (3.2) follows.

Now we prove (3.3). By the Bernstein inequality (4.1), we have

$$
\begin{aligned}
& \left(\frac{a_{m}}{m}\right)^{r}\left\|L_{m+2}^{*}\left(w, P_{M}\right)^{(r)} u\right\|_{p} \\
& \leq\left(\frac{a_{m}}{m}\right)^{r}\left\|\left[L_{m+2}\left(w, P_{M}\right)-L_{m+2}^{*}\left(w, P_{M}\right)\right]^{(r)} u\right\|_{p}+\left(\frac{a_{m}}{m}\right)^{r}\left\|P_{M}^{(r)} u\right\|_{p} \\
& \leq \mathrm{C}\left\|\left[L_{m+2}\left(w, P_{M}\right)-L_{m+2}^{*}\left(w, P_{M}\right)\right] u\right\|_{p}+\left(\frac{a_{m}}{m}\right)^{r}\left\|P_{M}^{(r)} u\right\|_{p} .
\end{aligned}
$$


We have already seen that the first addend is less than $\mathrm{Ce}^{-\mathrm{Am}}\|f u\|_{p}$, while, in order to estimate the second addend, we can use the inequality

$$
\left(\frac{a_{m}}{m}\right)^{r}\left\|P_{M}^{(r)} u\right\|_{p} \leq \mathrm{C}\left\{\omega^{r}\left(f, \frac{a_{m}}{m}\right)_{u, p}^{*}+\left(\frac{a_{m}}{m}\right)^{r}\|f u\|_{p}\right\},
$$

proved in 22]. This completes the proof.

Proof of Theorem 3.2. By the Remez-type inequality (4.3) we have

$$
\left\|L_{m+2}(w, f) u\right\|_{\infty} \leq \mathrm{C}\left\|L_{m+2}(w, f) u\right\|_{L^{\infty}\left(\mathcal{A}_{m}\right)} .
$$

For every $x \in \mathcal{A}_{m}$, we can write

$$
\begin{aligned}
&\left|L_{m+2}(w, f ; x)\right| u(x) \leq u(x) \sum_{|k| \leq \frac{m}{2}+1}\left|\ell_{k}^{*}(w ; x) f\left(x_{k}\right)\right| \\
& \leq\|f u\|_{\infty}\left\{\frac{\left|\ell_{d}^{*}(w ; x)\right| u(x)}{u\left(x_{d}\right)}+\sum_{k \neq d,|k| \leq \frac{m}{2}} \frac{\left|\ell_{k}^{*}(w ; x)\right| u(x)}{u\left(x_{k}\right)}\right. \\
&\left.\quad+\frac{\left|\ell_{\frac{m}{2}}^{*}+1(w ; x)\right| u(x)}{u\left(a_{m}\right)}+\frac{\left|\ell_{-\frac{m}{2}-1}^{*}(w ; x)\right| u(x)}{u\left(-a_{m}\right)}\right\} \\
&=:\|f u\|_{\infty}\left\{I_{1}+I_{2}+I_{3}+I_{4}\right\},
\end{aligned}
$$

where $x_{d} \in\left\{-x_{\frac{m}{2}}, \ldots, x_{\frac{m}{2}}\right\}$ is a zero closest to $x$.

By (4.9) and (4.12) we have

$$
I_{1}+I_{2} \leq \mathrm{C}\left[1+S_{m}(\alpha, \gamma, \mu, x)\right] \leq \mathrm{C} \log m,
$$

recalling the assumptions on $\alpha, \gamma, \mu$ and $x$.

Let us estimate only the term $I_{3}$, since $I_{4}$ can be handled in a similar way. By (4.11), since $\gamma-\frac{\alpha}{2} \geq 0$ and $0 \leq \mu \leq 1$, we have

$$
\frac{\left|\ell_{\frac{m}{2}+1}^{*}(w ; x)\right| u(x)}{u\left(a_{m}\right)} \leq \mathrm{C} \frac{v(|x|)}{v\left(a_{m}\right)}\left|\frac{x}{a_{m}}\right|^{\gamma-\frac{\alpha}{2}}\left(\frac{1+|x|}{1+a_{m}}\right)^{\mu} \leq \mathrm{C},
$$

$v$ being a nondecreasing function. Thus (3.5) and (3.6) remain proved.

Finally, by (3.2), inequality (3.7) follows from (3.5).

In the sequel we will need the following proposition.

Proposition 4.1. Let $t(x)=p_{m}(w ; x) \sqrt{w(x)} \sqrt[4]{a_{m}^{2}-x^{2}}$ and $g \in L^{p}, 1 \leq p<\infty$. Then we have

$$
\left(\int_{\mathbb{R}}|g(x) t(x)|^{p} \mathrm{~d} x\right)^{1 / p} \geq \mathrm{C}\left(\int_{-a_{m}}^{a_{m}}|g(x)|^{p} \mathrm{~d} x\right)^{1 / p}
$$

with $\mathrm{C} \neq \mathrm{C}(g, t)$.

Proof. Let $m$ be even. Set

$$
I_{m}=\bigcup_{|k| \leq \frac{m}{2}}\left(x_{k}-\frac{\delta}{8} \Delta x_{k}, x_{k}+\frac{\delta}{8} \Delta x_{k}\right),
$$

with a fixed $\delta>0$ sufficiently small and $x_{k}$ the zeros of $p_{m}(w)$. Moreover, define $C_{m}=\left(-a_{m}, a_{m}\right) \backslash I_{m}$. 
From (4.9) it follows that

$$
\left|p_{m}(w ; x)\right| \sqrt{w(x)} \sqrt[4]{a_{m}^{2}-x^{2}} \sim \frac{\left|x-x_{d}\right|}{\Delta x_{d}},
$$

where $x_{d}$ is a zero closest to $x$, and then we have

$$
|t(x)| \geq \mathrm{C}, \quad x \in C_{m} .
$$

Hence we get

$$
\begin{aligned}
\|g t\|_{p}^{p} & \geq \mathrm{C} \int_{C_{m}}|g(x)|^{p} \mathrm{~d} x \\
& =\mathrm{C}\left\{\int_{-a_{m}}^{a_{m}}|g(x)|^{p} \mathrm{~d} x+\int_{I_{m}}|g(x)|^{p} \mathrm{~d} x\right\} \\
& =\mathrm{C} a_{m}\left\{\int_{-1}^{1}\left|g\left(a_{m} y\right)\right|^{p} \mathrm{~d} y+\int_{I_{m}^{*}}\left|g\left(a_{m} y\right)\right|^{p} \mathrm{~d} y\right\},
\end{aligned}
$$

with

$$
I_{m}^{*}=\bigcup_{|k| \leq \frac{m}{2}}\left(\frac{x_{k}}{a_{m}}-\frac{\delta}{8} \frac{\Delta x_{k}}{a_{m}}, \frac{x_{k}}{a_{m}}+\frac{\delta}{8} \frac{\Delta x_{k}}{a_{m}}\right) .
$$

The measure of the subset $I_{m}^{*}$ is less than $\mathrm{C} \delta / 2$ and, by the absolute continuity of the integral, we can choose $\delta$ such that the integral extended to $I_{m}$ is one half of that extended to $[-1,1]$ and then we obtain (4.14).

Note that the same proof works if $m$ is odd.

In order to prove Lemma 3.3 we need the following result, proved in 27.

Lemma C. Denote by $x^{r, s}=|x|^{r}(1+|x|)^{s-r}$ and $1<p<\infty$. If $r \geq R, s \leq S$, $r>-\frac{1}{p}, s<1-\frac{1}{p}, R<1-\frac{1}{p}, S>-\frac{1}{p}$, then there exists a constant $\mathrm{C}$, independent of $f$, such that

$$
\int_{\mathbb{R}}\left|\int_{\mathbb{R}} \frac{f(y)}{x-y} x^{r, s} \mathrm{~d} y\right|^{p} \mathrm{~d} x \leq \mathrm{C} \int_{\mathbb{R}}\left|f(x) x^{R, S}\right|^{p} \mathrm{~d} x .
$$

Proof of Lemma 3.3. Let us first prove that (3.9) implies (3.8). By (4.3) and since $Q \in \mathcal{P}_{m+1}$, we have

$$
\begin{aligned}
\|Q u\|_{p} & \leq \mathrm{C}\|Q u\|_{L^{p}\left(\mathcal{A}_{m}\right)} \\
& =\mathrm{C} \sup _{\|g\|_{L^{q}\left(\mathcal{A}_{m}\right)}=1}\left|\int_{\mathcal{A}_{m}} Q(x) g(x) u(x) \mathrm{d} x\right| \\
& =\mathrm{C} \sup _{\|g\|_{L^{q}\left(\mathcal{A}_{m}\right)}=1}\left|\int_{\mathcal{A}_{m}} L_{m+2}^{*}(w, Q ; x) g(x) u(x) \mathrm{d} x\right| \\
& =\mathrm{C} \sup _{\|g\|_{L^{q}\left(\mathcal{A}_{m}\right)}=1}|A(g)|
\end{aligned}
$$

where $\frac{1}{p}+\frac{1}{q}=1$. 
Using (4.6) we get

$$
\begin{aligned}
|A(g)| & =\left|\sum_{|k| \leq j} \frac{Q\left(x_{k}\right) u\left(x_{k}\right)}{p_{m}^{\prime}\left(w ; x_{k}\right) u\left(x_{k}\right)\left(a_{m}^{2}-x_{k}^{2}\right)} \int_{\mathcal{A}_{m}} \frac{p_{m}(w ; x)\left(a_{m}^{2}-x^{2}\right) g(x) u(x)}{x-x_{k}} \mathrm{~d} x\right| \\
& \leq \frac{\mathrm{C}}{a_{m}^{3 / 2}} \sum_{|k| \leq j} \frac{\Delta x_{k}\left|Q\left(x_{k}\right)\right| u\left(x_{k}\right)\left|x_{k}\right|^{\frac{\alpha}{2}}-\gamma}{v\left(\left|x_{k}\right|\right)\left(1+\left|x_{k}\right|\right)^{\mu}}\left|\int_{\mathcal{A}_{m}} \frac{p_{m}(w ; x)\left(a_{m}^{2}-x^{2}\right) g(x) u(x)}{x-x_{k}} \mathrm{~d} x\right| \\
& \leq \frac{\mathrm{C}}{a_{m}^{3 / 2}} \sum_{|k| \leq j} \frac{\Delta x_{k}\left|Q\left(x_{k}\right)\right| u\left(x_{k}\right)\left|x_{k}\right|^{\frac{\alpha}{2}-\gamma}}{v\left(\left|x_{k}\right|\right)\left(1+\left|x_{k}\right|\right)^{\mu}}\left|\Pi\left(x_{k}\right)\right|,
\end{aligned}
$$

where

$$
\begin{gathered}
\Pi(y):=\int_{\mathcal{A}_{m}} \frac{\left(a_{m}^{2}-x^{2}\right) p_{m}(w ; x) R(x)-\left(a_{m}^{2}-y^{2}\right) p_{m}(w ; y) R(y)}{x-y} \frac{g(x) u(x)}{R(x)} \mathrm{d} x \\
=\mathcal{H}_{\mathcal{A}_{m}}(G ; y)-\Gamma(y) \mathcal{H}_{\mathcal{A}_{m}}\left(\frac{g u}{R} ; y\right), \\
G(y):=\left(a_{m}^{2}-y^{2}\right) p_{m}(w ; y) g(y) u(y), \\
\Gamma(y):=\left(a_{m}^{2}-y^{2}\right) p_{m}(w ; y) R(y),
\end{gathered}
$$

$R$ is an arbitrary polynomial of degree $\mathrm{bm}(\mathrm{b}>0)$, which will be specified in the sequel, and $\mathcal{H}_{\mathcal{A}_{m}}$ is the Hilbert transform extended to the subset $\mathcal{A}_{m}$. Here and in the sequel, for each set $A$, we denote by

$$
\mathcal{H}_{A}(f ; y)=\int_{A} \frac{f(x)}{x-y} \mathrm{~d} x \quad \text { and } \quad \mathcal{H}(f ; y)=\int_{\mathbb{R}} \frac{f(x)}{x-y} \mathrm{~d} x
$$

the Cauchy principal values of these integrals.

Note that $\Pi$ is a polynomial of degree at most $m+2+\mathrm{bm}$.

Using the Hölder inequality and Lemma 2.1 we get

$$
\begin{aligned}
|A(g)| \leq & \frac{\mathrm{C}}{a_{m}^{3 / 2}} S(Q)\left(\sum_{|k| \leq j} \Delta x_{k}\left[\frac{\left|x_{k}\right|^{\frac{\alpha}{2}-\gamma}}{v\left(\left|x_{k}\right|\right)\left(1+\left|x_{k}\right|\right)^{\mu}}\left|\Pi\left(x_{k}\right)\right|\right]^{q}\right)^{1 / q} \\
\leq & \frac{\mathrm{C}}{a_{m}^{3 / 2}} S(Q)\left\{\left(\int_{\mathcal{A}_{m}^{*}}\left[\frac{|y|^{\frac{\alpha}{2}-\gamma}}{v(|y|)(1+|y|)^{\mu}}\left|\mathcal{H}_{\mathcal{A}_{m}}(G ; y)\right|\right]^{q} \mathrm{~d} y\right)^{1 / q}\right. \\
& \left.+\left(\int_{\mathcal{A}_{m}^{*}}\left[\frac{|y|^{\frac{\alpha}{2}-\gamma}}{v(|y|)(1+|y|)^{\mu}}\left|\Gamma(y) \mathcal{H}_{\mathcal{A}_{m}}\left(\frac{g u}{R} ; y\right)\right|\right]^{q} \mathrm{~d} y\right)^{1 / q}\right\} \\
\leq & \frac{\mathrm{C}}{a_{m}^{3 / 2}} S(Q)\left\{\left(\int_{\mathcal{A}_{m}^{*}}\left[\frac{|y|^{\frac{\alpha}{2}-\gamma}}{v(|y|)(1+|y|)^{\mu}}\left|\mathcal{H}_{\mathcal{A}_{m}}(G ; y)\right|\right]^{q} \mathrm{~d} y\right)^{1 / q}\right. \\
& \left.+\left(\int_{\mathcal{A}_{m}^{*}}\left[\frac{|y|^{\frac{\alpha}{2}-\gamma}}{v(|y|)(1+|y|)^{\mu}}\left|\Gamma(y) \mathcal{H}_{\mathcal{A}_{m}}\left(\frac{g u}{R} ; y\right)\right|\right]^{q} \mathrm{~d} y\right)^{1 / q}\right\} \\
= & \frac{\mathrm{C}}{a_{m}^{3 / 2}} S(Q)\left\{B_{1}+B_{2}\right\},
\end{aligned}
$$


where

$$
S(Q):=\left(\sum_{|k| \leq j} \Delta x_{k}|Q u|^{p}\left(x_{k}\right)\right)^{1 / p},
$$

$\mathcal{A}_{m}^{*}:=\left[-\theta_{1} a_{m},-\mathrm{d} \frac{a_{m}}{m}\right] \cup\left[\mathrm{d} \frac{a_{m}}{m}, \theta_{1} a_{m}\right], \mathrm{d}$ is a fixed constant and $0<\theta<\theta_{1}<1$.

Using (4.5) we have

$$
|G(y)| \leq \mathrm{C} a_{m}^{3 / 2}|y|^{\gamma-\frac{\alpha}{2}} v(|y|)(1+|y|)^{\mu}|g(y)|
$$

for every $y \in \mathcal{A}_{m}$.

Setting $r=R=\frac{\alpha}{2}-\gamma$ and $s=S=\frac{\alpha}{2}-\gamma-\mu$, with $p$ replaced by $q$, and recalling (3.9), we can use Lemma C. Thus, by (4.16), we get

$$
\begin{aligned}
B_{1} & =\mathrm{C}\left(\int_{\mathcal{A}_{m}^{*}}\left[\frac{|y|^{\frac{\alpha}{2}-\gamma}}{v(|y|)(1+|y|)^{\mu}} \mid \mathcal{H}_{\mathcal{A}_{m}}(G ; y)\right]^{q} \mathrm{~d} y\right)^{1 / q} \\
& \leq \mathrm{C}\left(\int_{\mathbb{R}}\left[\frac{|y|^{\frac{\alpha}{2}-\gamma}}{v(|y|)(1+|y|)^{\mu}} \mid \mathcal{H}_{\mathcal{A}_{m}}(G ; y)\right]^{q} \mathrm{~d} y\right)^{1 / q} \\
& =\mathrm{C}\left(\int_{\mathbb{R}}\left[\frac{|y|^{\frac{\alpha}{2}-\gamma}}{v(|y|)(1+|y|)^{\mu}}\left|\mathcal{H}\left(\chi_{\mathcal{A}_{m}} G ; y\right)\right|\right]^{q} \mathrm{~d} y\right)^{1 / q} \\
& \leq \mathrm{C}\left(\int_{\mathcal{A}_{m}}\left[\frac{|y|^{\frac{\alpha}{2}-\gamma}}{v(|y|)(1+|y|)^{\mu}}|G(y)|\right]^{q} \mathrm{~d} y\right)^{1 / q} \\
& \leq \mathrm{C} a_{m}^{3 / 2}\left(\int_{\mathcal{A}_{m}}\left[\frac{|y|^{\frac{\alpha}{2}-\gamma}}{(1+|y|)^{\mu}}|y|^{\gamma-\frac{\alpha}{2}}(1+|y|)^{\mu}|g(y)|\right]^{q} \mathrm{~d} y\right)^{1 / q} \\
& \leq \mathrm{C} a_{m}^{3 / 2}\|g\|_{L^{q}\left(\mathcal{A}_{m}\right)}=\mathrm{C} a_{m}^{3 / 2}
\end{aligned}
$$

where $\chi_{\mathcal{A}_{m}}$ is the characteristic function of the set $\mathcal{A}_{m}$.

In order to estimate $B_{2}$ we choose $R \in \mathbb{P}_{\mathrm{bm}}$ (see for instance [11]) such that

$$
|R(y)| \sim \sqrt{w(y)}, \quad y \in \mathcal{A}_{m} .
$$

By (4.5) we have

$$
|\Gamma(y)| \leq \mathrm{C}\left(a_{m}^{2}-y^{2}\right)^{3 / 4} \frac{|R(y)|}{\sqrt{w(y)}} \leq \mathrm{C} a_{m}^{3 / 2} .
$$

Using the same arguments as in the estimate of $B_{1}$, by (4.18), we obtain

$$
\begin{aligned}
B_{2} & \leq \mathrm{C} a_{m}^{3 / 2}\left(\int_{\mathcal{A}_{m}}\left[\frac{|y|^{\frac{\alpha}{2}-\gamma}}{v(|y|)(1+|y|)^{\mu}}\left|\frac{g(y) u(y)}{R(y)}\right|\right]^{q} \mathrm{~d} y\right)^{1 / q} \\
& \leq \mathrm{C} a_{m}^{3 / 2}\left(\int_{\mathcal{A}_{m}}\left[\frac{|y|^{\frac{\alpha}{2}-\gamma}}{v(|y|)(1+|y|)^{\mu}}|y|^{\gamma-\frac{\alpha}{2}}(1+|y|)^{\mu}|g(y)|\right]^{q} \mathrm{~d} y\right)^{1 / q} \\
& \leq \mathrm{C} a_{m}^{3 / 2}\|g\|_{L^{q}\left(\mathcal{A}_{m}\right)}=\mathrm{C} a_{m}^{3 / 2} .
\end{aligned}
$$

Combining the estimates (4.17) and (4.19) with (4.15) and taking the supremum on $g$, we get (3.8).

It remains to prove that (3.8) implies (3.9). Since (3.8) holds true for every $Q \in \mathcal{P}_{m+1}$, in particular we have

$$
\left\|\ell_{1}^{*}(w) u\right\|_{p} \leq \mathrm{C}\left(\Delta x_{1}\right)^{1 / p} u\left(x_{1}\right)
$$


i.e.

$$
\mathrm{C} \geq \frac{x_{1}^{\frac{\alpha}{2}-\gamma}\left(\Delta x_{1}\right)^{1-\frac{1}{p}}}{\left(1+x_{1}\right)^{\mu}}\left(\left.\left.\int_{\mathbb{R}}\left|\frac{t(x)\left(a_{m}^{2}-x^{2}\right)}{\left(x-x_{1}\right)\left(a_{m}^{2}-x_{1}^{2}\right)}\right| x\right|^{\gamma-\frac{\alpha}{2}}(1+|x|)^{\mu}\right|^{p} \mathrm{~d} x\right)^{\frac{1}{p}},
$$

where $t(x)=p_{m}(w ; x) \sqrt{w(x)} \sqrt[4]{a_{m}^{2}-x^{2}}$.

For $x \in[0,1]$ we have $x_{1} \sim \Delta x_{1} \sim a_{m} / m$ and $a_{m}^{2}-x^{2} \sim a_{m}^{2}-x_{1}^{2}$. Using Proposition 4.1, it follows that

$$
\begin{aligned}
\mathrm{C} & \geq \mathrm{C} a_{m}^{\frac{\alpha}{2}-\gamma+1-\frac{1}{p}}\left(\int_{0}^{1}\left|\frac{t(x)}{x-x_{1}} x^{\gamma-\frac{\alpha}{2}}(1+x)^{\mu}\right|^{p} \mathrm{~d} x\right)^{1 / p} \\
& \geq \mathrm{C}\left(\frac{a_{m}}{m}\right)^{\frac{\alpha}{2}-\gamma+1-\frac{1}{p}}\left(\int_{0}^{1} x^{\left(\gamma-\frac{\alpha}{2}\right) p} \mathrm{~d} x\right)^{1 / p},
\end{aligned}
$$

since $\left|x-x_{1}\right| \leq 1$ and $1+x \geq 1$. Hence

$$
-\frac{1}{p}<\gamma-\frac{\alpha}{2} \leq 1-\frac{1}{p} \text {. }
$$

If $\gamma-\frac{\alpha}{2}=1-\frac{1}{p}$, then

$$
\begin{aligned}
\int_{0}^{1}\left|\frac{t(x)}{x-x_{1}} x^{\gamma-\frac{\alpha}{2}}\right|^{p} \mathrm{~d} x & >\int_{x_{2}}^{1} x^{\left(\gamma-\frac{\alpha}{2}-1\right) p} \mathrm{~d} x \\
& =\int_{x_{2}}^{1} \frac{\mathrm{d} x}{x} \sim \log \left(\frac{m}{a_{m}}\right) .
\end{aligned}
$$

Thus we get

$$
-\frac{1}{p}<\gamma-\frac{\alpha}{2}<1-\frac{1}{p}
$$

Moreover, by (4.20), we obtain

$$
\mathrm{C} \geq \mathrm{C}\left(\int_{a_{m} / 4}^{a_{m} / 2} x^{\left(\gamma-\frac{\alpha}{2}-1+\mu\right) p} \mathrm{~d} x\right)^{1 / p} \sim a_{m}^{\gamma-\frac{\alpha}{2}-1+\mu+\frac{1}{p}} ;
$$

then we must have $\gamma-\frac{\alpha}{2}-1+\mu+\frac{1}{p} \leq 0$. But, as before, we cannot have the equality; otherwise the integral would have order $\log a_{m}$. Therefore it must be

$$
\gamma-\frac{\alpha}{2}+\mu<1-\frac{1}{p} \text {. }
$$

This completes the proof.

Proof of Lemma 3.5. We first show that (3.9) implies (3.13) and (3.14). By Lemma 3.3 and using well-known arguments (see [26] for more details), we get

$$
\begin{aligned}
\left\|L_{m+2}^{*}(w, f) u\right\|_{p} & \leq \mathrm{C}\left(\sum_{|k| \leq j} \Delta x_{k}\left|f\left(x_{k}\right)\right|^{p} u^{p}\left(x_{k}\right)\right)^{1 / p} \\
& \leq \mathrm{C}\left\{\|f u\|_{L^{p}\left(\widetilde{A}_{m}\right)}+\left(\frac{a_{m}}{m}\right)^{1 / p} \int_{0}^{\frac{a_{m}}{m}} \frac{\Omega^{r}(f, t)_{u, p}^{*}}{t^{1+1 / p}} \mathrm{~d} t\right\},
\end{aligned}
$$

where $\widetilde{A}_{m}:=\left[-\theta a_{m},-\mathrm{d} \frac{a_{m}}{m}\right] \cup\left[\mathrm{d} \frac{a_{m}}{m}, \theta a_{m}\right], \mathrm{d}>0$. 
Let $P_{M} \in \mathbb{P}_{M}$ be a near best polynomial approximant of $f \in L_{u}^{p}$ and set $Q=$ $L_{m+2}^{*}\left(w, P_{M}\right)$. By (3.13) we get

$$
\begin{aligned}
& \left\|\left[f-L_{m+2}^{*}(w, f)\right] u\right\|_{p} \leq\|(f-Q) u\|_{p}+\left\|L_{m+2}^{*}(w, f-Q) u\right\|_{p} \\
& \leq \mathrm{C}\left\{\|(f-Q) u\|_{p}+\left(\frac{a_{m}}{m}\right)^{1 / p} \int_{0}^{\frac{a_{m}}{m}} \frac{\Omega^{r}(f-Q, t)_{u, p}^{*}}{t^{1+1 / p}} \mathrm{~d} t\right\} \\
& \leq \mathrm{C}\left\{\|(f-Q) u\|_{p}+\left(\frac{a_{m}}{m}\right)^{1 / p} \int_{0}^{\frac{a_{m}}{m}} \frac{\Omega^{r}(f, t)_{u, p}^{*}}{t^{1+1 / p}} \mathrm{~d} t+\left(\frac{a_{m}}{m}\right)^{r}\left\|Q^{(r)} u\right\|_{p}\right\},
\end{aligned}
$$

since, for any $g \in W_{r}^{p}(u), 1 \leq p \leq \infty$, if $\gamma+1 / p$ is not an integer, then we have (see [22])

$$
\omega^{r}(g, t)_{u_{p}}^{*} \leq \mathrm{C} t^{r}\left\|g^{(r)} u\right\|_{p}
$$

with $\mathrm{C} \neq \mathrm{C}(g, t)$.

Hence, using Lemma 3.1 and the Jackson-type inequality (2.2), inequality (3.14) follows from (4.21).

On the other hand, it is easily seen that (3.13) is equivalent to (3.14). Therefore it remains only to prove that (3.13) implies (3.9). To this end, let us consider the function

$$
f_{0}(x)= \begin{cases}0, & x \leq-x_{1}, \\ \frac{x}{2 x_{1}}+\frac{1}{2}, & -x_{1}<x \leq x_{1}, \\ -\frac{x}{\Delta x_{1}}+\frac{x_{2}}{\Delta x_{1}}, & x_{1}<x<x_{2}, \\ 0, & x \geq x_{2} .\end{cases}
$$

Thus we have $L_{m+2}^{*}\left(w, f_{0}\right)=\ell_{1}^{*}(w)$. By (3.13) we get

$$
\left\|L_{m+2}^{*}\left(w, f_{0}\right) u\right\|_{p} \leq \mathrm{C}\|u\|_{L^{p}\left(-x_{1}, x_{2}\right)},
$$

i.e.

$$
\left\|\ell_{1}^{*}(w) u\right\|_{p} \leq \mathrm{C}\left(\Delta x_{1}\right)^{1 / p} u\left(x_{1}\right),
$$

using the Marcinkiewicz-type inequality (3.8). As in the proof of Lemma 3.3 condition (3.9) follows from (4.23).

Proof of Theorem 3.6. We note that, using the Stechkin-type inequality (2.3), for every $f \in Z_{r}^{p}(u)$ we have

$$
\|f\|_{Z_{r}^{p}(u)} \sim\|f u\|_{p}+\sup _{i \geq 1}\left(\frac{i}{a_{i}}\right)^{r} E_{i}(f)_{u, p} .
$$

Therefore, for every $f \in Z_{s}^{p}(u), s \geq r>\frac{1}{p}$, we get

$$
\begin{aligned}
& \left\|f-L_{m+2}^{*}(w, f)\right\|_{Z_{r}^{p}(u)} \\
& =\left\|\left[f-L_{m+2}^{*}(w, f)\right] u\right\|_{p}+\sup _{i \geq 1}\left(\frac{i}{a_{i}}\right)^{r} E_{i}\left(f-L_{m+2}^{*}(w, f)\right)_{u, p} \\
& =: A+B .
\end{aligned}
$$

By using Lemma 3.5, we obtain

$$
A \leq \mathrm{C}\left(\frac{a_{m}}{m}\right)^{s}\|f\|_{Z_{s}^{p}(u)}
$$


Since

$$
E_{i}\left(f-L_{m+2}^{*}(w, f)\right)_{u, p} \begin{cases}\leq\left\|\left[f-L_{m+2}^{*}(w, f)\right] u\right\|_{p}, & i \leq m+1, \\ =E_{i}(f)_{u, p}, & i \geq m+2,\end{cases}
$$

then we have

$$
\begin{aligned}
B & =\sup _{i \geq 1}\left(\frac{i}{a_{i}}\right)^{r} E_{i}\left(f-L_{m+2}^{*}(w, f)\right)_{u, p} \\
& =\max \left\{\sup _{i \leq m+1}\left(\frac{i}{a_{i}}\right)^{r}\left\|\left[f-L_{m+2}^{*}(w, f)\right] u\right\|_{p}, \sup _{i \geq m+2}\left(\frac{i}{a_{i}}\right)^{r} E_{i}(f)_{u, p}\right\} \\
& \leq \max \left\{\mathrm{C}\left(\frac{m}{a_{m}}\right)^{r}\left(\frac{a_{m}}{m}\right)^{s}\|f\|_{Z_{s}^{p}(u)}, \sup _{i \geq m+2}\left(\frac{i}{a_{i}}\right)^{r-s}\left(\frac{i}{a_{i}}\right)^{s} E_{i}(f)_{u, p}\right\} \\
& \leq \mathrm{C}\left(\frac{a_{m}}{m}\right)^{s-r}\|f\|_{Z_{s}^{p}(u)} .
\end{aligned}
$$

Combining (4.25) and (4.26) with (4.24), we obtain (3.16) and (3.17).

Proof of Theorem 3.7. We first prove inequality (3.20). Assume $f \in W_{s}^{p}, s>r$, and set $Q_{m+1}=L_{m+2}^{*}(w, f)$. By (3.14) we have

$$
f-Q_{m+1}=\sum_{k=0}^{\infty}\left(Q_{2^{k+1}(m+1)}-Q_{2^{k}(m+1)}\right) \quad \text { a.e. }
$$

and, by the Bernstein inequality (4.1), it follows that

$$
\begin{aligned}
\left\|\left(f-Q_{m+1}\right)^{(r)} u\right\|_{p} & \leq \sum_{k=0}^{\infty}\left\|\left(Q_{2^{k+1}(m+1)}-Q_{2^{k}(m+1)}\right)^{(r)} u\right\|_{p} \\
& \leq \mathrm{C} \sum_{k=0}^{\infty}\left(\frac{2^{k+1}(m+1)}{a_{2^{k+1}(m+1)}}\right)^{r}\left\|\left[Q_{2^{k+1}(m+1)}-Q_{2^{k}(m+1)}\right] u\right\|_{p} .
\end{aligned}
$$

By (3.18) and taking into account that $a_{2^{k+1}(m+1)} \sim 2^{(k+1) / \lambda} a_{m}$, we get

$$
\begin{aligned}
\left\|\left(f-Q_{m+1}\right)^{(r)} u\right\|_{p} & \leq \mathrm{C}\|f\|_{W_{s}^{p}} \sum_{k=0}^{\infty}\left(\frac{a_{2^{k+1}(m+1)}}{2^{k+1}(m+1)}\right)^{s-r} \\
& \sim\left(\frac{a_{m}}{m}\right)^{s-r}\|f\|_{W_{s}^{p}} \sum_{k=0}^{\infty}\left(2^{(k+1)(1-\lambda) / \lambda}\right)^{s-r} \\
& \leq \mathrm{C}\left(\frac{a_{m}}{m}\right)^{s-r}\|f\|_{W_{s}^{p}} \sum_{k=0}^{\infty} 2^{(k+1)(1-\lambda) / \lambda} \\
& \leq \mathrm{C}\left(\frac{a_{m}}{m}\right)^{s-r}\|f\|_{W_{s}^{p}(u)},
\end{aligned}
$$

since $2^{(1-\lambda) / \lambda}<1$ for $\lambda>1$.

Now let us prove inequality (3.19). We have

$$
\left\|L_{m+2}^{*}(w, f)\right\|_{W_{r}^{p}(u)}=\left\|L_{m+2}^{*}(w, f) u\right\|_{p}+\left\|L_{m+2}^{*}(w, f)^{(r)} u\right\|_{p} .
$$

By (3.18) we get

$$
\left\|L_{m+2}^{*}(w, f) u\right\|_{p} \leq\|f u\|_{p}+\mathrm{C}\left(\frac{a_{m}}{m}\right)^{r}\|f\|_{W_{r}^{p}(u)} \leq \mathrm{C}\|f\|_{W_{r}^{p}(u)} .
$$


Let us consider $P_{M} \in \mathbb{P}_{M}$, with $M \sim m$ defined by (3.1), a near best polynomial approximant of $f \in W_{r}^{p}(u)$. We can write

$$
\left\|L_{m+2}^{*}(w, f)^{(r)} u\right\|_{p} \leq\left\|L_{m+2}^{*}\left(w, f-P_{M}\right)^{(r)} u\right\|_{p}+\left\|L_{m+2}^{*}\left(w, P_{M}\right)^{(r)} u\right\|_{p} .
$$

By (3.3) and (4.22) we have

$$
\left\|L_{m+2}^{*}\left(w, P_{M}\right)^{(r)} u\right\|_{p} \leq \mathrm{C}\|f\|_{W_{r}^{p}(u)},
$$

since $f \in W_{r}^{p}(u)$ and $\gamma+1 / p$ is not an integer.

Finally, by (4.1) and (4.28), we get

$$
\begin{aligned}
\left\|L_{m+2}^{*}\left(w, f-P_{M}\right)^{(r)} u\right\|_{p} & \leq \mathrm{C}\left(\frac{m}{a_{m}}\right)^{r}\left\|L_{m+2}^{*}\left(w, f-P_{M}\right) u\right\|_{p} \\
& \leq \mathrm{C}\left(\frac{m}{a_{m}}\right)^{r} E_{M}(f)_{u, p}+\mathrm{C}\left\|f-P_{M}\right\|_{W_{r}^{p}(u)} .
\end{aligned}
$$

Using Lemma 3.1 and the Jackson-type inequality (2.2), we obtain

$$
\left\|L_{m+2}^{*}\left(w, f-P_{M}\right)^{(r)} u\right\|_{p} \leq \mathrm{C}\|f\|_{W_{r}^{p}(u)} .
$$

By (4.28), (4.29) and (4.30), inequality (3.19) follows from (4.27).

Proof of Proposition 3.8, Let $P_{M} \in \mathbb{P}_{M}$, with $M$ given by (3.1) being a "near best polynomial approximant" of $f \in C_{\sigma}$. Then we can write

$$
\left|e_{m}(f)\right| \leq\left|e_{m}\left(f-P_{M}\right)\right|+\left|e_{m}\left(P_{M}\right)\right| .
$$

Since the ordinary Gaussian rule is exact for $P_{M}$, for the choice of $M$, by (4.4) and by the hypotheses, we get

$$
\begin{aligned}
\left|e_{m}\left(P_{M}\right)\right| & =\left|\sum_{|k|>j} \lambda_{k}(w) P_{M}\left(x_{k}\right)\right| \\
& \leq\left\|P_{M} \sigma\right\|_{L^{\infty}\left\{|x|>\theta a_{m}(w)\right\}} \sum_{|k|>j} \frac{\lambda_{k}(w)}{\sigma\left(x_{k}\right)} \\
& \leq\left\|P_{M} \sigma\right\|_{L^{\infty}\left\{|x|>(1+\theta) a_{M}(\sigma)\right\}} \int_{\mathbb{R}} \frac{w(x)}{\sigma(x)} \mathrm{d} x \\
& \leq \mathrm{Ce}^{-\mathrm{A} m}\left\|P_{M} \sigma\right\|_{\infty} \leq \mathrm{Ce}^{-\mathrm{A} m}\|f \sigma\|_{\infty} .
\end{aligned}
$$

Moreover, we have

$$
\begin{aligned}
\left|e_{m}\left(f-P_{M}\right)\right| & \leq\left\|\left(f-P_{M}\right) \sigma\right\|_{\infty}\left(\int_{\mathbb{R}} \frac{w(x)}{\sigma(x)} \mathrm{d} x+\sum_{|k| \leq j} \frac{\lambda_{k}(w)}{\sigma\left(x_{k}\right)}\right) \\
& \leq \mathrm{C} E_{M}(f)_{\sigma, \infty} .
\end{aligned}
$$

Hence, by (4.31), (4.32) and (4.33), we get (3.22).

Proof of Theorem 3.9. First of all we remark that (3.27) is equivalent to

$$
\sup _{y \in \mathbb{R}} \sup _{m}\left\|L_{m+2}^{*}(w, f) k_{y} u\right\|_{1} \leq \mathrm{C}\|f u\|_{\infty}
$$

where $k_{y}(x):=k(x, y)$. 
Let us prove that (3.25) and (3.26) imply (4.34). By definitions (3.23) and (3.24) we can write

$$
\begin{aligned}
\left|G_{m}(f, y)\right| & =\left\|L_{m+2}^{*}(w, f) k_{y} u\right\|_{1} \\
& \leq\left\|L_{m+2}^{*}(w, f) k_{y} u\right\|_{L^{1}\left(I_{m}\right)}+\left\|L_{m+2}^{*}(w, f) k_{y} u\right\|_{L^{1}\left(\mathbb{R} \backslash I_{m}\right)} \\
& =: A_{1}+A_{2},
\end{aligned}
$$

where $I_{m}=\left[-a_{m}, a_{m}\right]$.

Using arguments and notation similar to those in the proof of Lemma 3.3. we get

$$
A_{1} \leq \mathrm{C} \frac{\|f u\|_{\infty}}{a_{m}^{3 / 2}} \sum_{|k| \leq j} \frac{\left|x_{k}\right|^{\frac{\alpha}{2}-\gamma} \Delta x_{k}}{\left(1+\left|x_{k}\right|\right)^{\mu}}\left|\Pi\left(x_{k}\right)\right|,
$$

where

$$
\begin{aligned}
\Pi(t) & :=\mathcal{H}_{I_{m}}\left(G^{*} ; t\right)-\Gamma^{*}(t) \mathcal{H}_{I_{m}}\left(\frac{g k_{y} u}{R} ; t\right), \\
G^{*}(x) & :=\left(a_{m}^{2}-x^{2}\right) p_{m}(w ; x) g(x) k_{y}(x) u(x), \\
\Gamma^{*}(t) & :=\left(a_{m}^{2}-t^{2}\right) p_{m}(w ; t) R(t),
\end{aligned}
$$

$g(x):=\operatorname{sgn}\left\{L_{m+2}^{*}(w, f ; x) k_{y}(x)\right\}, R$ is an arbitrary polynomial of degree $\mathrm{b} m$ (b > 0 ), which will be specified in the sequel and $\mathcal{H}_{I_{m}}$ is the Hilbert transform extended to $I_{m}$.

By the Marcinkiewicz-type inequality (2.12), we have

$$
A_{1} \leq \mathrm{C} \frac{\|f u\|_{\infty}}{a_{m}^{3 / 2}} \int_{I_{m}} \frac{|t|^{\frac{\alpha}{2}-\gamma}}{(1+|t|)^{\mu}}|\Pi(t)| \mathrm{d} t .
$$

Moreover, by (3.25), it follows that

$$
\begin{aligned}
A_{1} & \leq \mathrm{C} \frac{\|f u\|_{\infty}}{a_{m}^{3 / 2}} \int_{I_{m}}|\Pi(t)| \mathrm{d} t \\
& \leq \mathrm{C} \frac{\|f u\|_{\infty}}{a_{m}^{3 / 2}}\left\{\int_{I_{m}}\left|\mathcal{H}_{\mathcal{A}_{m}}\left(G^{*} ; t\right)\right| \mathrm{d} t+\int_{I_{m}}\left|\Gamma^{*}(t)\right|\left|\mathcal{H}_{I_{m}}\left(\frac{g k_{y} u}{R}, t\right)\right| \mathrm{d} t\right\} \\
& =\mathrm{C} \frac{\|f u\|_{\infty}}{a_{m}^{3 / 2}}\left\{B_{1}+B_{2}\right\} .
\end{aligned}
$$

In order to estimate $B_{1}$, we can write

$$
G^{*}(x)=\left(a_{m}^{2}-x^{2}\right)^{3 / 4} \sqrt[4]{a_{m}^{2}-x^{2}} p_{m}(w ; x) g(x) k_{y}(x) u(x)=: a_{m}^{3 / 2} G_{1}(x),
$$

with $G_{1} \in L \log ^{+} L$, and by (4.5) we have

$$
\left|G_{1}(x)\right| \leq \mathrm{C}|x|^{\gamma-\frac{\alpha}{2}}(1+|x|)^{\mu}\left|k_{y}(x)\right| .
$$

Therefore, using a well-known result (see for instance [32]), we get

$$
\begin{aligned}
B_{1} & =\mathrm{C} a_{m}^{3 / 2} \int_{I_{m}}\left|\mathcal{H}_{I_{m}}\left(G_{1}, t\right)\right| \mathrm{d} t \\
& \leq \mathrm{C} a_{m}^{3 / 2} \int_{I_{m}}\left|G_{1}(t)\right|\left(1+\log ^{+}\left|G_{1}(t)\right|\right) \mathrm{d} t \\
& \leq \mathrm{C} a_{m}^{3 / 2} \int_{\mathbb{R}}|t|^{\gamma-\frac{\alpha}{2}}(1+|t|)^{\mu}\left|k_{y}(t)\right|\left(1+\log ^{+}\left|k_{y}(t)\right|+\log ^{+}|t|\right) \mathrm{d} t .
\end{aligned}
$$


By (3.26) the previous integral is bounded.

The term $B_{2}$ can be estimated in the same way. In fact, choosing $R$ such that $R(x) \sim \sqrt{w(x)}$ for $x \in I_{m}$, we obtain

$$
\left|\Gamma^{*}(t)\right| \leq \mathrm{C} a_{m}^{3 / 2}
$$

and

$$
B_{2} \leq \mathrm{C} a_{m}^{3 / 2} \int_{I_{m}}\left|k_{y}(x)\right| \frac{u(x)}{\sqrt{w(x)}}\left(1+\log ^{+}\left|k_{y}(x)\right|+\log ^{+}|x|\right) \mathrm{d} x .
$$

From the estimates (4.36) and (4.37), it follows that

$$
A_{1} \leq \mathrm{C}\|f u\|_{\infty} .
$$

Concerning the term $A_{2}$, we can write

$$
\begin{aligned}
A_{2} & =\left\|L_{m+2}^{*}(w, f) k_{y} u\right\|_{L^{1}\left(J_{\delta, m}\right)}+\left\|L_{m+2}^{*}(w, f) k_{y} u\right\|_{L^{1}\left(\left\{|x|>a_{m}(1+\delta)\right\}\right)} \\
& =: D_{1}+D_{2},
\end{aligned}
$$

where $J_{\delta, m}:=\left\{a_{m}<|x|<a_{m}(1+\delta)\right\}$.

By virtue of (4.6), $D_{1}$ can be estimated as

$$
D_{1} \leq \mathrm{C} \frac{\|f u\|_{\infty}}{a_{m}^{3 / 2}} \sum_{|k| \leq j} \frac{\left|x_{k}\right|^{\frac{\alpha}{2}-\gamma} \Delta x_{k}}{\left(1+\left|x_{k}\right|\right)^{\mu}} \int_{J_{\delta, m}} \frac{\left|p_{m}(w ; x)\left(a_{m}^{2}-x^{2}\right) k_{y}(x)\right| u(x)}{x-x_{k}} \mathrm{~d} x .
$$

Moreover, by inequalities (4.3), for $p=\infty$, and (4.5), we get

$$
\begin{aligned}
D_{1} & \leq \mathrm{C} \frac{\|f u\|_{\infty}}{a_{m}} \sum_{|k| \leq j} \Delta x_{k} \int_{J_{\delta, m}}\left|k_{y}(x)\right||x|^{\gamma-\frac{\alpha}{2}}(1+|x|)^{\mu} \mathrm{d} x \\
& \leq \mathrm{C}\|f u\|_{\infty} \int_{\mathbb{R}}\left|k_{y}(x)\right||x|^{\gamma-\frac{\alpha}{2}}(1+|x|)^{\mu} \mathrm{d} x \leq \mathrm{C}\|f u\|_{\infty},
\end{aligned}
$$

since $x-x_{k} \geq(1-\theta) a_{m}$ and $\left|x^{2}-a_{m}^{2}\right|^{3 / 4} \leq \mathrm{C} a_{m}^{3 / 2}$, and using (3.25).

Let us estimate $D_{2}$. By (4.4) and (4.13), for some $\tau>0$, we have

$$
\begin{aligned}
D_{2} & \leq \mathrm{Ce}^{-\mathrm{A} m}\left\|L_{m+2}^{*}(w, f) u\right\|_{\infty}\left\|k_{y}\right\|_{1} \\
& \leq \mathrm{Ce}^{-\mathrm{A} m} m^{\tau}\|f u\|_{\infty}\left\|k_{y}\right\|_{1} \leq \mathrm{C}\|f u\|_{\infty} .
\end{aligned}
$$

It follows that

$$
A_{2} \leq \mathrm{C}\|f u\|_{\infty} .
$$

Combining (4.38) and (4.39) in (4.35), and taking the supremum over all $y \in \mathbb{R}$ we get (3.27).

Finally, in order to show that (4.34) implies (3.28), let us consider a function $f_{0}$ such that $\left|f_{0}(x)\right| \leq 1$ for every $x \in \mathbb{R}, f_{0}\left(x_{k}\right)=\operatorname{sgn}\left[p_{m}^{\prime}\left(w ; x_{k}\right)\left(x-x_{k}\right)\right]$ for $x_{1} \leq x_{k} \leq 1$ and $f_{0}\left(x_{k}\right)=0$ for $x_{k}>1$ and for $x_{k}<x_{1}$. Hence we have

$$
L_{m+2}^{*}\left(w, f_{0} ; x\right)=\sum_{x_{1} \leq x_{k} \leq 1} \frac{p_{m}(w ; x)\left(a_{m}^{2}-x^{2}\right)}{\left|p_{m}^{\prime}\left(w ; x_{k}\right)\right|\left|x-x_{k}\right|\left(a_{m}^{2}-x_{k}^{2}\right)} .
$$


Using (4.6), for $x \in[0,1]$, we obtain

$$
\begin{aligned}
\left|L_{m+2}^{*}(w, f ; x)\right| & =\left|p_{m}(w ; x)\right| \sum_{x_{1} \leq x_{k} \leq 1}\left(\frac{a_{m}^{2}-x^{2}}{a_{m}^{2}-x_{k}^{2}}\right) \frac{1}{\left|p_{m}^{\prime}\left(w ; x_{k}\right)\right|\left|x-x_{k}\right|} \\
& \sim\left|p_{m}(w ; x)\right| \sqrt[4]{a_{m}^{2}-x^{2}} \sum_{x_{1} \leq x_{k} \leq 1} \Delta x_{k} \sqrt{w\left(x_{k}\right)} \\
& \geq\left|p_{m}(w ; x)\right| \sqrt[4]{a_{m}^{2}-x^{2}} \sum_{x_{1} \leq x_{k} \leq 1} \int_{x_{k}}^{x_{k+1}} \sqrt{w(t)} \mathrm{d} t \\
& \geq\left|p_{m}(w ; x)\right| \sqrt[4]{a_{m}^{2}-x^{2}} \int_{1 / 2}^{1} \sqrt{w(t)} \mathrm{d} t \\
& =\mathrm{C}\left|p_{m}(w ; x)\right| \sqrt[4]{a_{m}^{2}-x^{2}} .
\end{aligned}
$$

By (4.34), for every $y \in \mathbb{R}$, we have

$$
\begin{aligned}
\|u\|_{\infty} & \geq \mathrm{C} \int_{\mathbb{R}}\left|L_{m+2}^{*}\left(w, f_{0} ; x\right) k(x, y)\right| u(x) \mathrm{d} x \\
& \geq \mathrm{C} \int_{0}^{1}\left|p_{m}(w ; x) k(x, y)\right| \sqrt[4]{a_{m}^{2}-x^{2}} u(x) \mathrm{d} x \\
& \geq \mathrm{C} \int_{0}^{1}|k(x, y)| \frac{u(x)}{\sqrt{w(x)}} \mathrm{d} x,
\end{aligned}
$$

using Proposition 4.1, Since

$$
\int_{\mathbb{R} \backslash[0,1]}|k(x, y)| \frac{u(x)}{\sqrt{w(x)}} \mathrm{d} x<\infty \quad \forall y \in \mathbb{R},
$$

taking the supremum over all $y$, we get (3.28).

\section{REFERENCES}

[1] M. C. De Bonis, G. Mastroianni \& M. G. Russo, Polynomial approximation with special doubling weights, Acta Sci. Math. (Szeged) 69 (2003), 159-184. MR.1991663 (2004f:41002)

[2] M. C. De Bonis, G. Mastroianni \& M. Viggiano, K-functionals, moduli of smoothness and weighted best approximation on the semiaxis, Functions, series, operators (Budapest, 1999), János Bolyai Math. Soc., Budapest 2002, pp. 181-211. MR.1981564 (2004d:41009)

[3] B. Della Vecchia \& G. Mastroianni, Gaussian rules on unbounded intervals, J. Complexity 19 (2003), 247-258. MR1984112(2004c:41061)

[4] A. S. Cvetković \& G. V. Milovanović, The Mathematica package "OrthogonalPolynomials", Facta Univ. Ser. Math. Inform. 19 (2004), 17-36. MR2122752 (2006i:33038)

[5] T. Kasuga \& R. Sakai, Orthonormal polynomials with generalized Freud-type weights, J. Approx. Theory 121 (2003), no. 1, 13-53. MR1962994 (2004f:42040)

[6] T. Kasuga \& R. Sakai, Orthonormal polynomials for generalized Freud-type weights and higher-order Hermite-Fejér interpolation polynomials, J. Approx. Theory 127 (2004), no. 1, 1-38. MR2053531 (2005e:42074)

[7] T. Kasuga \& R. Sakai, Conditions for uniform or mean convergence of higher order HermiteFejér interpolation polynomials with generalized Freud-type weights, Far East J. Math. Sci. (FJMS) 19 (2005), no. 2, 145-199. MR2188311 (2006g:42003)

[8] O. Kis, Lagrange interpolation with nodes at the roots of Sonin-Markov polynomials (in Russian), Acta Math. Acad. Sci. Hungar. 23 (1972), 389-417. MR0312118 (47:680)

[9] C. Laurita \& G. Mastroianni, Condition numbers in numerical methods for Fredholm integral equations of the second kind, J. Integral Equations Appl. 14 (2002), no. 3, 311-341. MR:1982158 (2004c:65171)

[10] C. Laurita \& G. Mastroianni, $L^{p}$-Convergence of Lagrange interpolation on the semiaxis, Acta Math. Hungarica 120 (2008), no. 3, 249-273. MR2429523 (2009d:41005) 
[11] A. L. Levin \& D. S. Lubinsky, Christoffel functions, orthogonal polynomials, and Nevai's conjecture for Freud weights, Constr. Approx. 8 (1992), no. 4, 463-535. MR.1194029 (94f:42030)

[12] E. Levin \& D. S. Lubinsky, Orthogonal polynomials for exponential weights, CMS Books in Mathematics/Ouvrages de Mathématiques de la SMC, vol. 4, Springer-Verlag, New York, 2001. MR1840714 (2002k:41001)

[13] E. Levin \& D. Lubinsky, Orthogonal polynomials for exponential weights $x^{2 \rho} \mathrm{e}^{-2 Q(x)}$ on $[0, d)$, J. Approx. Theory 134 (2005), no. 2, 199-256. MR2142299 (2006a:42037)

[14] D. S. Lubinsky, A survey of weighted polynomial approximation with exponential weights, Surv. Approx. Theory 3 (2007), 1-105. MR2276420 (2007k:41001)

[15] D. S. Lubinsky \& G. Mastroianni, Mean convergence of extended Lagrange interpolation with Freud weights, Acta Math. Hungar. 84 (1999), no. 1-2, 47-63. MR1696546 (2000g:41007)

[16] D. S. Lubinsky \& G. Mastroianni, Converse quadrature sum inequalities for Freud weights. II, Acta Math. Hungar. 96 (2002), no. 1-2, 147-168. MR1912494 (2003c:41040)

[17] G. Mastroianni \& G. Monegato, Truncated quadrature rules over $(0, \infty)$ and Nyström-type methods, SIAM J. Numer. Anal. 41 (2003), no. 5, 1870-1892. MR2035010 (2005b:65022)

[18] G. Mastroianni \& D. Occorsio, Markov-Sonin Gaussian rule for singular functions, J. Comput. Appl. Math. 169 (2004), no. 1, 197-212. MR2071270(2005e:65026)

[19] G. Mastroianni \& D. Occorsio, Mean convergence of Fourier sums on unbounded intervals, Studia Univ. "Babes-Bolyai" Mathematica LII (2007), no. 4, 89-103. MR2429342 (2009e:42063)

[20] G. Mastroianni \& M. G. Russo, Lagrange interpolation in weighted Besov spaces, Constr. Approx. 15 (1999), 257-289. MR1669080 (2000b:41001)

[21] G. Mastroianni \& J. Szabados, Polynomial approximation on infinite intervals with weights having inner zeros, Acta Math. Hungar. 96 (2002), no. 3, 221-258. MR.1919162 (2003f:41007)

[22] G. Mastroianni \& J. Szabados, Direct and converse polynomial approximation theorems on the real line with weights having zeros, N.K. Govil, H.N. Mhaskar, R.N. Mohpatra, Z. Nashed and J. Szabados, eds. Frontiers in Interpolation and Approximation, Dedicated to the memory of A. Sharma, Boca Raton, Florida, Taylor \& Francis Books, 2006, pp. 287-306. MR 2274182 (2007j:41004)

[23] G. Mastroianni \& V. Totik, Jackson type inequalities for doubling and $A_{p}$ weights, Supplemento ai rendiconti del Circolo Matematico di Palermo, Proceedings of the Third International Conference on Functional Analysis and Approximation Theory, Acquafredda di Maratea (Potenza, Italy), September 23-28, Volume I, 1996, pp. 83-99. MR1644543 (99g:41013)

[24] G. Mastroianni \& V. Totik, Jackson type inequalities for doubling weights. II, East J. Approx. 5 (1999), 101-116. MR 1688386 (2000c:41018)

[25] G. Mastroianni \& V. Totik, Best approximation and moduli of smoothness for doubling weights, J. Approx Theory 110 (2001), 180-199. MR.1830536 (2002g:41044)

[26] G. Mastroianni \& P. Vértesi, Fourier sums and Lagrange interpolation on $(0,+\infty)$ and $(-\infty,+\infty)$, N.K. Govil, H.N. Mhaskar, R.N. Mohpatra, Z. Nashed and J. Szabados, eds. Frontiers in Interpolation and Approximation, Dedicated to the memory of A. Sharma, Boca Raton, Florida, Taylor \& Francis Books, 2006, pp. 307-344. MR2274183 (2007g:42006)

[27] B. Muckenhoupt, Mean convergence of Hermite and Laguerre series. II, Trans. Amer. Math. Soc. 147 (1970), 433-460. MR0256051 (41:711)

[28] P. G. Nevai, Orthogonal polynomials on the real line associated with the weight $|x|^{\alpha} \mathrm{e}^{-|x|^{\beta}}$. I, (in Russian) Acta Math. Acad. Sci. Hungar. 24 (1973), 335-342. MR0330559 (48:8896)

[29] P. G. Nevai, Mean convergence of Lagrange interpolation. II, J. Approx. Theory 30 (1980), no. 4, 263-276. MR616953 (82i:41003)

[30] D. Occorsio \& M. G. Russo, The $L^{p}$-weighted Lagrange interpolation on Markov-Sonin zeros, Acta Math. Hungar. 112 (2006), no. 1-2, 57-84. MR2251130 (2007e:41002)

[31] E. B. Saff \& V. Totik, Logarithmic potentials with external fields, A series of Comprehensive Studies in Mathematics, vol. 316, Springer-Verlag, Berlin, 1997. MR.1485778 (99h:31001)

[32] E. Stein, Singular integrals and differentiability properties of functions, Princeton Univ. Press, Princeton, N. J., 1970. MR0290095 (44:7280)

[33] J. Szabados, Weighted Lagrange and Hermite-Fejér interpolation on the real line, J. Inequal. Appl. 1 (1997), no. 2, 99-123. MR1731425 (2000h:41010)

[34] P. Vértesi, An Erdös-type convergence process in weighted interpolation. I. Freud-type weights, Acta Math. Hungar. 91 (2001), no. 3, 195-215. MR1913026 (2003g:41003) 
Dipartimento di Matematica e Informatica, Università degli Studi della Basilicata, V.Le Dell'Ateneo Lucano 10, I-85100 Potenza, Italy

E-mail address: mastroianni.csafta@unibas.it

Dipartimento di Matematica e Informatica, Università degli Studi della Basilicata, V.le Dell'Ateneo Lucano 10, I-85100 Potenza, Italy

E-mail address: incoronata.notarangelo@unibas.it 